\title{
Polarimetric SAR time series change analysis over agricultural areas
}

\author{
Alberto Alonso-González, Member, IEEE, Carlos López-Martínez, Senior Member, IEEE, \\ Konstantinos P. Papathanassiou, Fellow, IEEE and Irena Hajnsek, Fellow, IEEE
}

\begin{abstract}
This paper proposes a change detection and analysis technique for monitoring the phenological development of agricultural vegetation by means of multi-temporal Polarimetric Synthetic Aperture Radar (PolSAR) acquisitions. The technique relies on the generalized eigendecomposition of the polarimetric covariance matrices of the individual acquisitions. It both quantifies the magnitude of the change between PolSAR images acquired at different times as well as provides an interpretation of occurred change in terms of the modified polarization states. This makes the algorithm suitable for investigating scattering dynamics associated with the phenological development of agricultural vegetation. To aid the interpretation of the changes detected, a representation based on the polarization states affected by the change process is proposed. The technique is evaluated using part of the multi-temporal AGRISAR 2006 campaign data set. This data set consists of 12 quad-polarimetric images acquired by the German Aerospace Center (DLR) E-SAR airborne system at L-band from April to August 2006 over the Demmin test site. It covers large parts of the development cycle of different crop types. As part of the evaluation, reference ground measurements are used to facilitate the interpretation of the data. The evaluation focus on 5 important crop types: wheat, barley, rape, maize and sugar beet. The results show that the proposed technique is able to detect and characterize different types of changes related to distinct development states of different crop types as the plant growing, maturation and drying processes.
\end{abstract}

Index Terms-SAR, Polarimetry, Change analysis, Change detection, Time series, Agriculture, Crop monitoring

\section{INTRODUCTION}

$\mathbf{S}$ YNTHETIC Aperture Radar (SAR) configurations are active imaging systems that operate in the microwave range of the electromagnetic spectrum. They are able to provide high resolution images of the radar reflectivity across large scenes [1]. Moreover, since the atmosphere is almost transparent at these frequencies, SAR systems are able to acquire imagery independently of the weather conditions or the day-night cycle. Furthermore, when operated on a space-borne platform, they can achieve global coverage with a regular temporal resolution. In this sense, the increasing presence of space-borne SAR sensors has facilitated the construction of temporal series data sets. These data sets contain different acquisitions of the same scene at different times. This information is of considerable

A. Alonso-González, K. P. Papathanassiou and I. Hajnsek are with the Microwaves and Radar Institute, German Aerospace Center (DLR), 82234 Wessling, Germany (e-mail: alberto.alonso-gonzalez@dlr.de).

I. Hajnsek is also with the Institute of Environmental Engineering, Swiss Federal Institute of Technology (ETH) Zürich, 8092 Zurich, Switzerland.

C. López-Martínez is with the Signal Theory and Communications Department (TSC), Universitat Politècnica de Catalunya (UPC), 08034 Barcelona, Spain. value, as it allows to monitor the temporal evolution or development of the scene. This trend is expected to continue in the future, as current and future missions focus on the systematic coverage of the Earth surface at short repeat pass intervals. Relevant examples include the ESA Sentinel-1 or the future JAXA ALOS-4 and DLR Tandem-L missions. All this makes SAR systems a valuable source of information for the monitoring of Earth's environment.

SAR configurations are capable of achieving high spatial resolution by a coherent processing of the radar echoes received by a moving platform. Even for high resolution systems, however, the size of the resolution cell is much larger than the wavelength. The received echo for each resolution cell is given by the coherent combination of the responses of all the individual scatterers, and depends on their distribution within the cell, which is generally unknown. As a consequence, in the absence of a dominant scatterer, the resulting image has a noisy appearance called speckle [2]. Speckle phenomenon complicates the interpretation of SAR data, especially in the case of change detection, as it hinders a reliable estimation of the target response. Numerous speckle reduction techniques have been proposed, which tend to improve the estimates by adapting to the content of the image. The employed methodologies range from a simple set of directional windows [3] to a more complex adaptive neighborhood [4] or a region-based approach [5]. Additionally, some other non-local approaches have also been proposed as [6], for instance.

Polarimetric SAR (PolSAR) systems provide additional information by exploiting the vectorial nature of the electromagnetic waves. When two orthogonal polarization states are employed in transmission and reception, polarization synthesis may be applied in order to explore the target response in the complete polarimetric domain [7][8]. During the last decade, PolSAR has been effectively employed for extracting useful biophysical and geophysical information of the scene, which makes it very valuable and convenient for monitoring the Earth surface [9][10][11].

Several methods have been proposed to aid in the interpretation of the obtained polarimetric response for the different areas of the scene. In this context, polarimetric decompositions are often used to decompose the target response information into a reduced set of parameters. Several polarimetric decompositions have been proposed, for instance: Huynen [12], Krogager [13], Cameron [14], Freeman and Durden [15] or Touzi [16] decompositions. One of the most popular decompositions, proposed by Cloude and Pottier [17], is based on the projection of the coherence matrix into the orthogonal 
polarimetric basis generated by its eigenvectors. A set of three parameters, Entropy $(H)$, Anisotropy $(A)$ and mean Alpha angle $(\bar{\alpha})$ are obtained, which may be employed to interpret the scattering process. In this case Entropy indicates the power distribution of the different scattering processes, Anisotropy indicates the dominance of the second eigenvalue versus the third one, in terms of the reflected power, and the mean Alpha angle refers to the type of scattering [17].

Well established approaches also exist for PolSAR change detection, as [18][19], where a test statistic is employed to estimate the probability of change of the different areas of the image. Other techniques have proposed a similar concept in a region-based approach, as described in [20]. Although all these techniques detect the amount of change between two images, none of them provide information concerning the type of change. The change interpretation may be performed by analyzing a polarimetric decomposition of both images, in order to see which parameters differ. However, this information is not complete, as only a reduced set of parameters are obtained from the complete polarimetric space. Additionally, these decompositions describe the scattering for each of the images, not the change between them, which could cover up some of the changes. This paper proposes a fully polarimetric change analysis and decomposition technique to detect and analyze the changes between two images. A change representation for the identification and interpretation of the changes is proposed. This representation is generalized to time series, where it can be used to determine the principal changes on the development of different crop types.

The content of this paper is structured as follows: Section II introduces the basics of SAR Polarimetry, focusing on the target description and its statistics. Section III analyzes different ways of detecting the changes between two PolSAR images and describes the proposed polarimetric change analysis and the corresponding change representation. In Section IV, the suggested technique is evaluated with a real data set over agricultural areas, focusing on the detection of the different crop changes over time. The results are compared with insitu collected measurements and photographs to evaluate the detected changes. Finally, Section V presents the conclusions.

\section{SAR POLARIMETRY}

A PolSAR system measures the scattering matrix $\mathbf{S}$ for each resolution cell [21][8]. Under the backscattering alignment convention, $\mathbf{S}$ may be vectorized into the scattering vector $\mathbf{k}$ in the Pauli basis [22] as

$$
\mathbf{k}=\frac{1}{\sqrt{2}}\left[\begin{array}{lll}
S_{h h}+S_{v v}, & S_{h h}-S_{v v}, & S_{h v}+S_{v h}
\end{array}\right]^{T}
$$

where $S_{i j}$ represents the complex scattering coefficient for the received and transmitted polarization combination $i, j \in[h, v]$, $h$ and $v$ denote horizontal and vertical polarization, respectively, and ${ }^{T}$ stands for vector transpose.

If the number of individual scattering elements within the resolution cell is sufficiently large, then the Central Limit Theorem applies [23][24]. In this case, $\mathbf{k}$ follows a zero-mean complex Gaussian distribution

$$
p_{\mathbf{k}}(\mathbf{k})=\frac{1}{\pi^{3}|\mathbf{T}|} \exp \left(-\mathbf{k}^{H} \mathbf{T}^{-1} \mathbf{k}\right)
$$

where ${ }^{H}$ represents the complex hermitian transpose and $\mathbf{T}$ is the polarimetric coherency matrix, i.e. the covariance matrix in the Pauli basis, that parameterizes the distribution

$$
\mathbf{T}=E\left\{\mathbf{k k}^{H}\right\},
$$

$E\{x\}$ denotes the statistical expectation of the stochastic process $\mathrm{x}$ and $|\cdot|$ is the matrix determinant operator.

The polarimetric response is usually described by the sample coherency matrix $\mathbf{Z}$ which corresponds to the Maximum Likelihood Estimator (MLE) of T. Under the Gaussian hypothesis, it completely characterizes the polarimetric response [25]

$$
\mathbf{Z}=\left\langle\mathbf{k} \mathbf{k}^{H}\right\rangle_{n}=\frac{1}{n} \sum_{i=1}^{n} \mathbf{k}_{i} \mathbf{k}_{i}^{H}
$$

where $\mathbf{k}_{i}$ represents the scattering vector of the $i$-th pixel and $n$ represents the number of independent pixels averaged.

Note that this estimation is only valid when averaging homogeneous samples, i.e. samples with the same statistical distribution. In this case, the estimated sample coherency matrix $\mathbf{Z}$ is Wishart distributed, characterized by the distribution [25]

$$
p_{\mathbf{Z}}(\mathbf{Z})=\frac{n^{p n}|\mathbf{Z}|^{n-p}}{|\mathbf{T}|^{n} \widetilde{\Gamma}_{p}(n)} \operatorname{etr}\left(-n \mathbf{T}^{-1} \mathbf{Z}\right)
$$

where $\operatorname{etr}(\mathbf{X})$ is the exponential of the trace of matrix $\mathbf{X}$ and

$$
\widetilde{\Gamma}_{p}(n)=\pi^{\frac{1}{2} p(p-1)} \prod_{i=1}^{p} \Gamma(n-i+1) .
$$

where $p=3$ is the dimension of the $\mathbf{k}$ vector and $\Gamma$ is the gamma function.

Note that the Wishart distribution defined in (5) is only valid for $n \geq 3$. For $n<3$ the sample coherency matrix $\mathbf{Z}$ is singular, i.e. with a rank below 3 and $|\mathbf{Z}|=0$. Therefore, some degree of averaging or speckle filtering is required for an appropriate polarimetric analysis.

\section{Polarimetric CHANGE DETECTION AND ANALYSiS}

In the following, the sample coherency matrix $\mathbf{Z}$ will be employed assuming the validity of the Gaussian hypothesis for distributed scattering processes. The detection and analysis of changes between two polarimetric images of the same scene acquired at different times is based on the corresponding sample coherency matrices $\mathbf{Z}_{1}$ and $\mathbf{Z}_{2}$.

\section{A. Polarimetric change detection}

The sample coherency matrix follows a Wishart distribution as described in (5). This can be used to assess whether the two observed coherency matrices $\mathbf{Z}_{1}$ and $\mathbf{Z}_{2}$ correspond to the same distribution. This approach was employed in [18] in order to derive a Wishart test statistic $Q$

$$
Q=2^{6 n} \frac{\left|\mathbf{Z}_{1}\right|^{n}\left|\mathbf{Z}_{2}\right|^{n}}{\left|\mathbf{Z}_{1}+\mathbf{Z}_{2}\right|^{2 n}}
$$

where both sample coherency matrices $\mathbf{Z}_{1}$ and $\mathbf{Z}_{2}$ are assumed to be obtained by averaging the same number of samples $n$. Usually, the logarithmic test statistic $-\ln Q$ is employed, 
which has a large value when $\mathbf{Z}_{1}$ and $\mathbf{Z}_{2}$ belong to two different distributions and a small value otherwise. As long as $\mathbf{Z}_{1}$ and $\mathbf{Z}_{2}$ belong to the same distribution, it is assumed that no changes have occurred. On the other hand, a change in the scene will induce a change in the coherency matrix distribution.

Alternatively, the geometry of the hermitian positive definite matrix cone may be used to define a similarity measure, that is, a geodesic measure adapted to the coherency matrix space [26]. This idea was employed in [20] to define a change measure across $N$ acquisitions. Applying this measure to a pair of acquisitions, given by $\mathbf{Z}_{1}$ and $\mathbf{Z}_{2}$, the same $d_{g}$ measure as defined in [26] is obtained

$$
d_{g}=\left\|\log \left(\mathbf{z}_{1}^{-1 / 2} \mathbf{Z}_{2} \mathbf{Z}_{1}^{-1 / 2}\right)\right\|_{F}
$$

where $\log (\mathbf{X})$ refers to the matrix logarithm and $\|\mathbf{X}\|_{F}$ represents the Frobenius matrix norm of $\mathbf{X}$. As for the test statistic above, this will yield lower values when $\mathbf{Z}_{1}$ and $\mathbf{Z}_{2}$ are similar to each other, indicating no change, and a large value otherwise.

\section{B. Polarimetric change analysis}

The measures defined in (7) and (8) may be employed to obtain an idea of the amount of change between $\mathbf{Z}_{1}$ and $\mathbf{Z}_{2}$ but they do not give information related to the type of change in the polarimetric space or the direction of change (i.e. increase or decrease).

One of the first approaches to analyze the change between two coherency matrices $\mathbf{Z}_{1}$ and $\mathbf{Z}_{2}$, was to employ the polarimetric contrast measure $P_{c}$ at a given polarization state w [27][28]

$$
P_{c}\left(\mathbf{Z}_{1}, \mathbf{Z}_{2}, \mathbf{w}\right)=\frac{\mathbf{w}^{H} \mathbf{Z}_{2} \mathbf{w}}{\mathbf{w}^{H} \mathbf{Z}_{1} \mathbf{w}} .
$$

This measure may be interpreted as the ratio between the backscattered power at both acquisitions for the polarization state $\mathbf{w}$. Since it is a ratio of two powers, $P_{c}$ is always real and positive. $1<P_{c}<\infty$ indicates an increase in backscattered power from $\mathbf{Z}_{1}$ to $\mathbf{Z}_{2}$ at the polarization state $\mathbf{w}$, whereas $0<P_{c}<1$ represents a decrease. When $P_{c}=1$ there is no change.

The maximum (minimum) polarimetric contrast can be addressed in terms of the optimum polarization state $\mathbf{w}_{\text {opt }}$ which maximizes (or minimizes) $P_{c}$ in (9). The solution for this optimization problem is related to the generalized eigenvalues of $\mathbf{Z}_{1}$ and $\mathbf{Z}_{2}$ [27][8]

$$
\mathbf{Z}_{2} \mathbf{w}=\lambda \mathbf{Z}_{1} \mathbf{w}
$$

which may be obtained as the solution of the equation

$$
\left|\mathbf{Z}_{2}-\lambda \mathbf{Z}_{1}\right|=0 .
$$

The generalized eigenvalues $\lambda_{i}$ (for $i=1 \ldots 3$ ) define the $P_{c}$ values that are obtained according to (9) using the corresponding generalized eigenvectors $\mathbf{w}_{i}$. Sorted in descending order such that $\lambda_{1} \geq \lambda_{2} \geq \lambda_{3}>0, \lambda_{1}$ corresponds to the maximum and $\lambda_{3}$ to the minimum $P_{c}$, while $\lambda_{2}$ to an intermediate value.
If $\mathbf{Z}_{1}$ is invertible, then from (10) follows

$$
\mathbf{Z}_{1}^{-1} \mathbf{Z}_{2} \mathbf{w}=\lambda \mathbf{w}
$$

the classical eigenvalue problem for $\mathbf{Z}_{1}^{-1} \mathbf{Z}_{2}$ with the characteristic equation

$$
\left|\mathbf{Z}_{1}^{-1} \mathbf{Z}_{2}-\lambda \mathbf{I}\right|=0 .
$$

It is worth noticing that $\lambda_{i}$ and $\mathbf{w}_{i}$ depend on both covariance matrices $\mathbf{Z}_{1}$ and $\mathbf{Z}_{2}$. They represent only the relative changes between them, according to the ratio defined in (9). Since the $\mathbf{Z}_{1}^{-1} \mathbf{Z}_{2}$ matrix is in general not hermitian, the generalized eigenvectors $\mathbf{w}_{i}$ are not orthogonal to each other, unlike the eigenvectors of $\mathbf{Z}_{1}$ and $\mathbf{Z}_{2}$. If both matrices are not singular, then the set of $\mathbf{w}_{i}$ vectors conform a non-orthogonal basis for the complete polarimetric space [29][30].

The relation between the generalized eigenvalues and the polarimetric contrast was already analyzed in [31] for polarimetric change detection, where the generalized eigenvalues $\lambda_{i}$ were used to obtain the magnitude of the change. Here the information of the generalized eigenvectors $\mathbf{w}_{i}$ is exploited for the interpretation of the type of change. This interpretation is based on the fact that any unitary vector can be associated to a scattering matrix, i.e. a rank 1 scatterer [32][8]. While the obtained $\mathbf{w}_{i}$ are not restricted by orthogonality, it is important to note that this restriction $\mathbf{w}_{i}^{H} \mathbf{w}_{j}=0$ for $i \neq j$ is not broken, but replaced by [29]

$$
\begin{array}{rlrl}
\mathbf{w}_{i}^{H} \mathbf{Z}_{2} \mathbf{w}_{j}=0 & & \forall i \neq j \\
\mathbf{w}_{i}^{H} \mathbf{Z}_{1} \mathbf{w}_{j}=0 & \forall i \neq j .
\end{array}
$$

In fact, the generalized eigenvectors $\mathbf{w}_{i}$ conform a nonorthogonal basis that simultaneously diagonalizes both $\mathbf{Z}_{1}$ and $\mathbf{Z}_{2}$ hermitian matrices [33]. Formally, they correspond to the projection vectors that maximize and minimize (9).

When a temporal series of $N$ coherency matrices $\mathbf{Z}_{1}, \mathbf{Z}_{2}, \ldots, \mathbf{Z}_{N}$ acquired at different times is available, the change analysis can be performed between every acquisition pair $\mathbf{Z}_{i}, \mathbf{Z}_{j}$. In this case, the obtained change contributions are interconnected. For instance, when considering 3 acquisitions, the change from the first to the second and from the second to the third acquisition is obtained from the eigendecomposition of the matrices $\mathbf{M}_{1 \rightarrow 2}=\mathbf{Z}_{1}^{-1} \mathbf{Z}_{2}$ and $\mathbf{M}_{2 \rightarrow 3}=\mathbf{Z}_{2}^{-1} \mathbf{Z}_{3}$, as described in (12). This also implies that

$$
\mathbf{Z}_{2}=\mathbf{Z}_{1} \mathbf{M}_{1 \rightarrow 2}
$$

so that the change between the first and the third acquisitions $\mathbf{M}_{1 \rightarrow 3}$ is related to $\mathbf{M}_{1 \rightarrow 2}$ and $\mathbf{M}_{2 \rightarrow 3}$ as

$$
\begin{aligned}
\mathbf{Z}_{3} & =\mathbf{Z}_{2} \mathbf{M}_{2 \rightarrow 3}=\mathbf{Z}_{1} \mathbf{M}_{1 \rightarrow 2} \mathbf{M}_{2 \rightarrow 3} \\
\mathbf{M}_{1 \rightarrow 3} & =\mathbf{Z}_{1}^{-1} \mathbf{Z}_{3}=\mathbf{M}_{1 \rightarrow 2} \mathbf{M}_{2 \rightarrow 3} .
\end{aligned}
$$

In general, for each $i, j$ pair such that $j>i$, it can be seen that $\mathbf{Z}_{j}=\mathbf{Z}_{i} \mathbf{M}_{i \rightarrow j}$ where

$$
\mathbf{M}_{i \rightarrow j}=\mathbf{Z}_{i}^{-1} \mathbf{Z}_{j}=\prod_{k=i}^{j-1} \mathbf{M}_{k \rightarrow k+1} .
$$

For this reason, this change analysis technique is referred to as multiplicative change analysis [31][34][35], in contrast to the additive change analysis [36], where changes are defined in an additive context $\mathbf{Z}_{2}=\mathbf{Z}_{1}+\mathbf{C}_{1 \rightarrow 2}$. 


\section{Polarimetric change representation}

Following the discussion above, the generalized eigenvalues and eigenvectors can be used to represent the change between $\mathbf{Z}_{1}$ and $\mathbf{Z}_{2}$. To this end, the generalized eigenvectors $\mathbf{w}_{i}$ normalized such that $\left\|\mathbf{w}_{i}\right\|=1$ and expressed in the Pauli basis are weighted by the associated generalized eigenvalues $\lambda_{i}$ in $\mathrm{dB}$ [34]. Additionally, the changes are separated into increasing $\left(\lambda_{i}>1\right)$ and decreasing $\left(\lambda_{i}<1\right)$ in terms of power ratio. In this way, two 3 -dimensional real vectors $\mathbf{p}_{\text {inc }}$ and $\mathbf{p}_{\text {dec }}$ comprising the magnitude of the Pauli elements of $\mathbf{w}_{i}$ weighted by their significance are obtained; $\mathbf{p}_{\text {inc }}$ associated with the increasing and $\mathbf{p}_{d e c}$ with the decreasing scattering contributions. Accordingly, each element $k$ of $\mathbf{p}_{\text {inc }}$ and $\mathbf{p}_{\text {dec }}$, $p_{i n c}^{k}$ and $p_{d e c}^{k}$, is defined as [34][35]

$$
\begin{aligned}
& p_{\text {inc }}^{k}=\left[\sum_{i \mid \lambda_{i}>1}^{p}\left(10 \log _{10} \lambda_{i} \cdot\left|w_{i}^{k}\right|\right)^{2}\right]^{\frac{1}{2}} \\
& p_{\text {dec }}^{k}=\left[\sum_{i \mid \lambda_{i}<1}^{p}\left(-10 \log _{10} \lambda_{i} \cdot\left|w_{i}^{k}\right|\right)^{2}\right]^{\frac{1}{2}},
\end{aligned}
$$

which can also be expressed in vector notation as

$$
\begin{aligned}
& \mathbf{p}_{\text {inc }}=\left[\sum_{i \mid \lambda_{i}>1}^{p}\left(10 \log _{10} \lambda_{i}\right)^{2} \cdot \mathbf{w}_{i} \odot \mathbf{w}_{i}^{*}\right]^{\odot \frac{1}{2}} \\
& \mathbf{p}_{\text {dec }}=\left[\sum_{i \mid \lambda_{i}<1}^{p}\left(-10 \log _{10} \lambda_{i}\right)^{2} \cdot \mathbf{w}_{i} \odot \mathbf{w}_{i}^{*}\right]^{\odot \frac{1}{2}},
\end{aligned}
$$

where $\odot$ represents the element by element product (Hadamard product) and $\mathbf{a}^{\odot \frac{1}{2}}$ represent the element by element square root of vector $\mathbf{a}$.

Even if $\mathbf{p}_{\text {inc }}$ and $\mathbf{p}_{d e c}$ contain only a subset of the information provided by the generalized eigenvalue decomposition in (10), since the complex phases between different polarimetric channels are ignored, they still provide a useful visualization of the magnitude and type of change occurred. In terms of a RGB representation, it combines the (increasing and decreasing) change information for all the $\lambda_{i}$ and $\mathbf{w}_{i}$ into two images where the amount of change is represented by the color brightness while the color itself indicates the type of change.

\section{EXPERIMENTAL RESULTS}

For the evaluation of the proposed technique, a subset of the data acquired in the frame of the AgriSAR 2006 campaign [37] is used. The campaign took place from April to August 2006 on the Demmin test site located in northern Germany. Demmin is mainly an agricultural site with fields of different crop types. During this time, DLR's airborne E-SAR sensor acquired almost every two weeks fully polarimetric SAR data at L-band as well as dual-pol and single-pol data at C- and Xband, respectively. Parallel to the SAR acquisitions a large set of ground measurements including the documentation of the phenological development stage, vegetation height and plant

\begin{tabular}{ccc}
\hline \hline Acq. Date & Heading & Notes \\
\hline $19 / 04 / 2006$ & $270^{\circ}$ & Intensive ground measurements \\
$20 / 04 / 2006$ & $203^{\circ}$ & Intensive ground measurements \\
$03 / 05 / 2006$ & $270^{\circ}$ & \\
$11 / 05 / 2006$ & $270^{\circ}$ & \\
$16 / 05 / 2006$ & $270^{\circ}$ & \\
$24 / 05 / 2006$ & $270^{\circ}$ & \\
$06 / 06 / 2006$ & $203^{\circ}$ & Intensive ground measurements \\
$07 / 06 / 2006$ & $270^{\circ}$ & Intensive ground measurements \\
$13 / 06 / 2006$ & $270^{\circ}$ & \\
$21 / 06 / 2006$ & $270^{\circ}$ & \\
$05 / 07 / 2006$ & $270^{\circ}$ & Intensive ground measurements \\
$06 / 07 / 2006$ & $203^{\circ}$ & Intensive ground measurements \\
$12 / 07 / 2006$ & $270^{\circ}$ & \\
$26 / 07 / 2006$ & $270^{\circ}$ & \\
$02 / 08 / 2006$ & $270^{\circ}$ & \\
\hline \hline
\end{tabular}

TABLE I

LIST OF E-SAR ACQUISITIONS OVER THE DEMMIN TESTSITE IN THE AgriSAR 2006 CAMPAign. All ACQUisitions WERE COLLECTED AT X-, C- AND L-BAND IN SINGLE-, DUAL- AND QUAD-POL, RESPECTIVELY

water content as well as soil moisture has been collected at every date. On the three "intensive" campaign dates, i.e. the 18-20th of April, the 5-8th of June and the 3-8th of July 2006, a higher sampling of ground measurements was performed and supplementary information was collected. On the intensive campaign dates the SAR data acquisition was also performed along two different headings $\left(270^{\circ}\right.$ and $\left.203^{\circ}\right)$. The list of SAR acquisitions and the dates corresponding to the intensive ground measurements are summarized in Table I. In the following the $12 \mathrm{~L}$-band acquisitions performed at a single heading $\left(270^{\circ}\right)$ will be used for the change analysis evaluation.

\section{A. Results of the three intensive measurement acquisitions}

We start with the change analysis between the three intensive campaign dates. In order to reduce the effect of speckle and to obtain a reliable estimation of the polarimetric response, the Temporal Evolution Binary Partition Tree (TE BPT) with a pruning threshold of $\delta_{p}=-2 d B$ has been employed, as described in [20].

The Pauli RGB images of these three acquisitions are shown in Fig. 1 while Fig. 2 shows the corresponding land-use map where the different colors represent the different crop types. Note that the large variation of the incidence angle my be observed from near- to far-range typical for airborne acquisitions (in our case from 25 to 55 degrees) entails the dependence of the scattering mechanisms on the angle of incidence: even for the same crop type a significant change in the polarimetric signature from near to far range may occur.

The change of the vegetation height and plant water content during the campaign is shown in Fig. 3, where the three intensive campaign dates are marked by a red rectangle. For the evaluation of the phenological stage of the individual crops, the $\mathrm{BBCH}$ scale (Biologische Bundesanstalt Bundessortenamt und CHemische Industrie) [38] is used. The BBCH scale differentiates between 10 main growth stages (indexed from 0 to 9 ). Within each main growth stage further 10 secondary development stages (indexed as well from 0 to 9) are differen- 


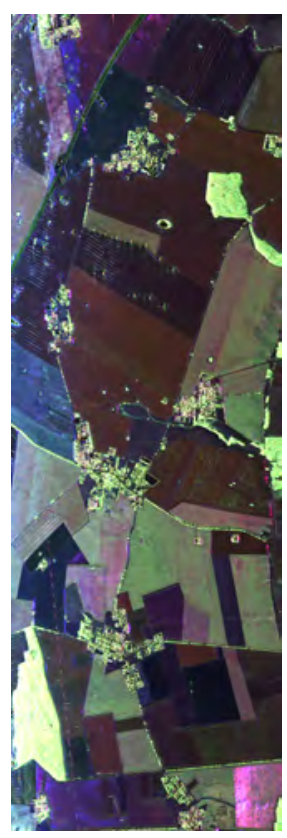

(a) 19th April

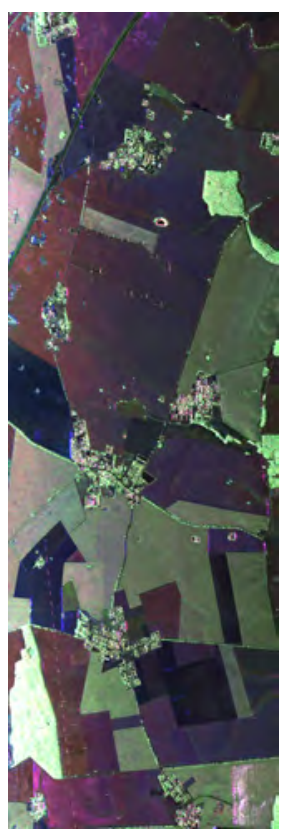

(b) 7th June

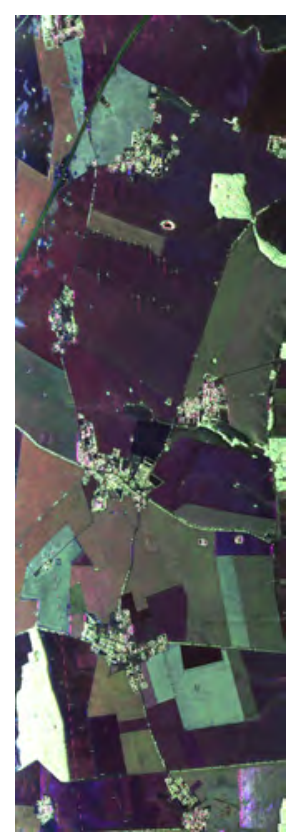

(c) 5th July
Fig. 1. Pauli RGB of the three L-band acquisitions of the AgriSAR 2006 campaign processed with the Temporal Evolution BPT $\left(\delta_{p}=-2 d B\right)$.

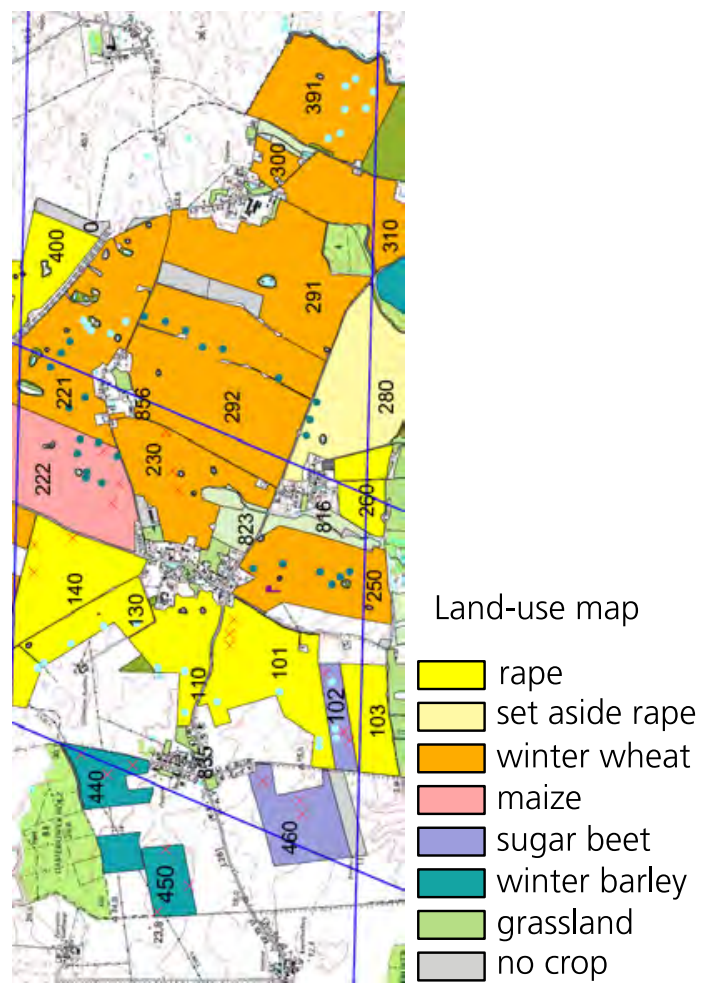

Fig. 2. Land-use map of the imaged area.

tiated so that each field is denoted by an index ranging from 00 to 99 , characterizing its phenological stage:

00-09: Germination

10-19: Leaf development

20-29: Formation of side shots / tillering

30-39: Stem elongation or rosette growth

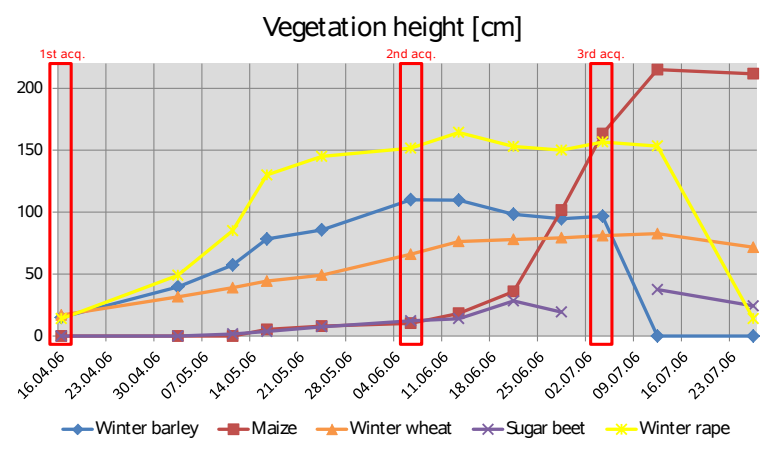

(a) Vegetation height

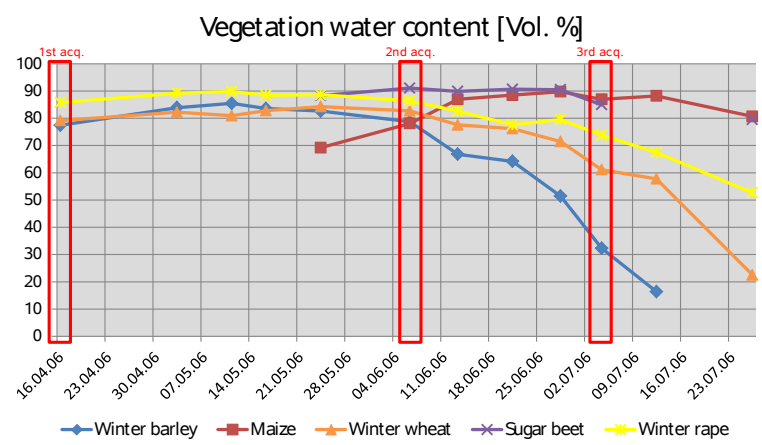

(b) Plant water content

Fig. 3. Vegetation height and water content evolution for different types of crops.

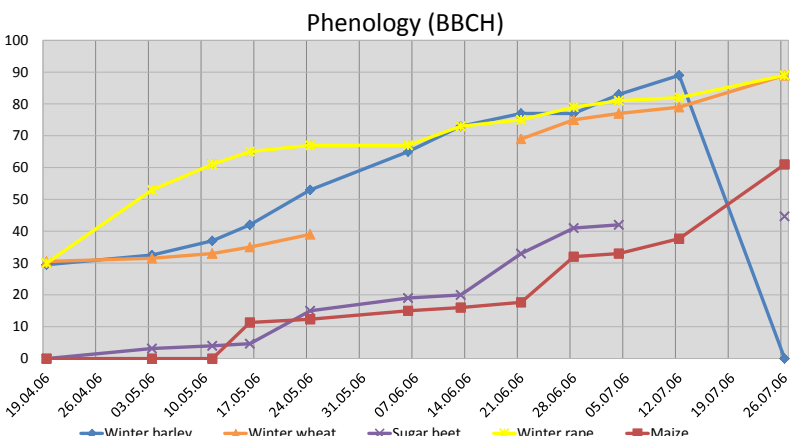

Fig. 4. Phenology evolution measured from ground according to the $\mathrm{BBCH}$ scale.

40-49: Development of harvestable vegetative plant parts or vegetatively propagated organs / booting

50-59: Inflorescence emergence / heading

60-69: Flowering

70-79: Development of fruit

80-89: Ripening or maturity of fruit and seed

90-99: Senescence

Fig. 4 shows the $\mathrm{BBCH}$ scale for the crop types in the site during the campaign.

The change analysis between the three "intensive" campaign dates are shown in Fig. 5. Fig. 5a shows the results of the Wishart test statistic $-\ln Q$ measure, with $Q$ defined in (7), for the acquisitions on the 19th of April and the 7th of June. Additionally, Figs $5 \mathrm{~b}$ and $5 \mathrm{c}$ show the change according to the $d_{g}$ measure defined in (8) between the 19th of April and the 
7 th of June and between the 7th of June and the 5th of July.

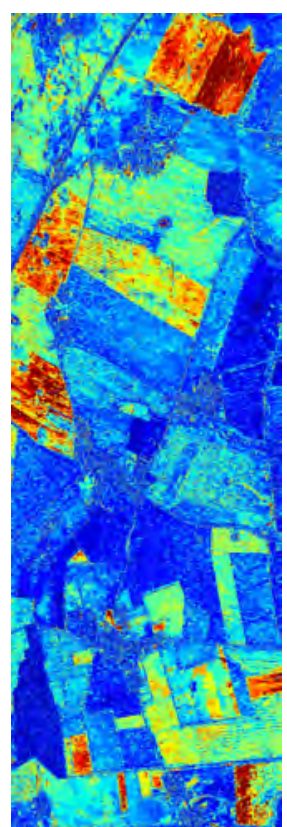

(a) $-\ln Q, 19 / 4-7 / 6$

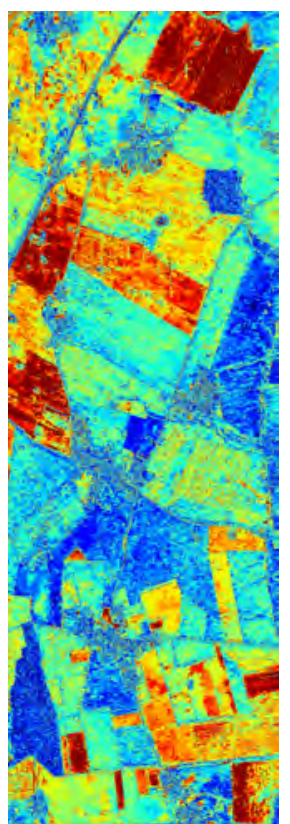

(b) $d_{g}, 19 / 4-7 / 6$

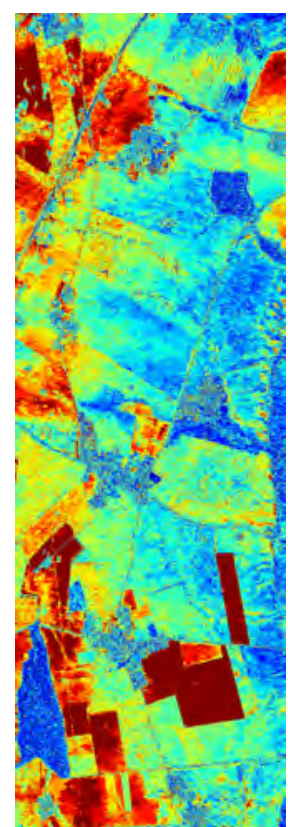

(c) $d_{g}, 7 / 6-5 / 7$

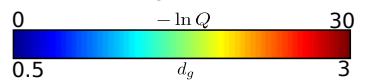

Fig. 5. Wishart test statistic $-\ln Q$ (7) measure (a) and geodesic similarity measure $d_{g}(8)$ (b) change detection maps for the acquisition pair 19th April - 7th June, and $d_{g}$ for acquisition pair 7th June - 5th July (c). No change is represented in blue and a large change in red.

For both change metrics a larger value indicates a bigger change, appearing in reddish colors. On the other hand, bluish regions represent areas that have not changed significantly.

As mentioned in Section I, polarimetric decomposition techniques may be employed to interpret the scattering occurring in each of the acquisitions [39]. For comparison the Entropy $(H)$ and the mean Alpha angle $(\bar{\alpha})$ [17] for the three acquisitions are shown in Fig. 6. Some of the changes detected in Fig. 5 may be also depicted when looking on the change of $H$ and $\bar{\alpha}$, but many others not. This is specially the case between the second and the third acquisition, where the mean Alpha angle remains the same over most of the fields and only small changes of the entropy are visible. As a result, the detection and interpretation of changes may not be easy using these parameters. This is due to the fact that they rather provide information related to the type of scattering at each acquisition, than about its change between acquisitions.

In order to obtain the amount and type of change, the polarimetric change analysis proposed in Section III-B is applied. The generalized eigenvalues for the acquisitions on the 19th of April and the 7th of June are shown in Fig. 7. The eigenvalues are sorted $\left(\lambda_{1} \geq \lambda_{2} \geq \lambda_{3}\right)$ and scaled in $\mathrm{dB}$. No change corresponds to $0 \mathrm{~dB}$, while the increase or decrease appear with positive or negative values, respectively. The Pauli RGB representations of the associated generalized eigenvectors are shown in Fig. 8.

Comparing the change detection results in Fig. 5b, with the corresponding generalized eigenvalues, in Fig. 7, makes

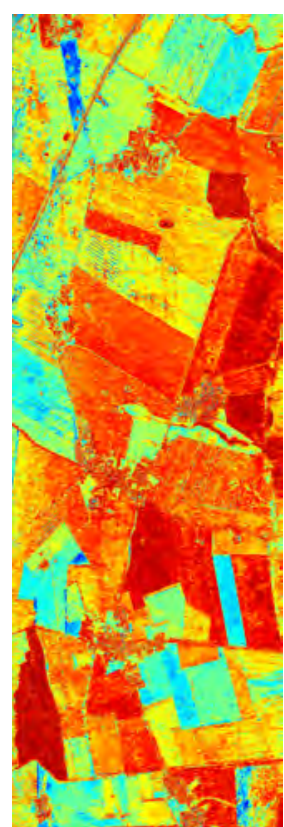

(a) $H$ 19th April

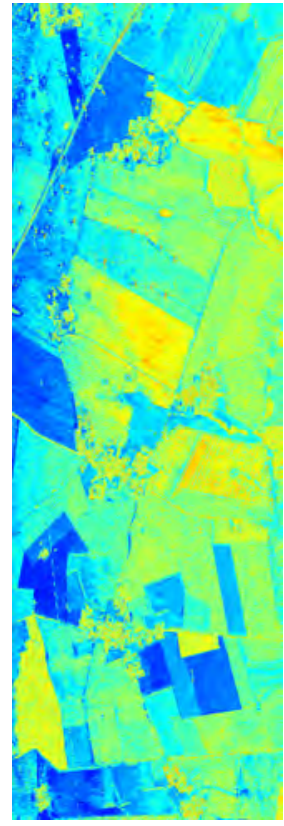

(d) $\bar{\alpha}$ 19th April

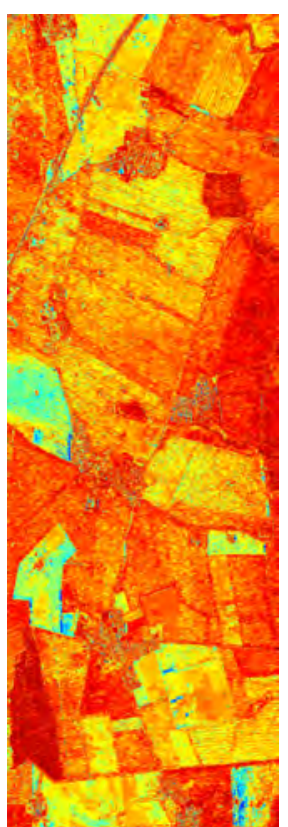

(b) $H$ 7th June

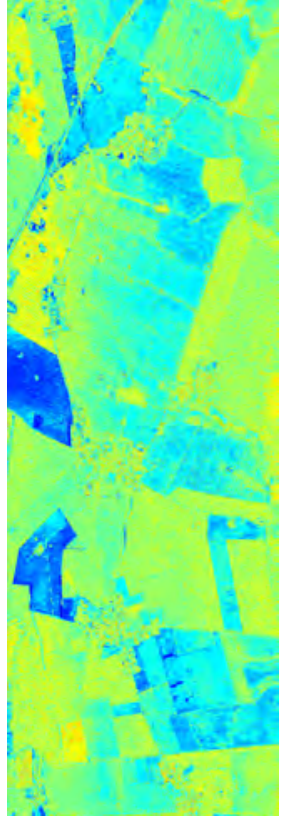

(e) $\bar{\alpha} 7$ th June

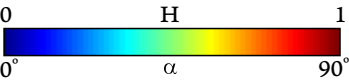

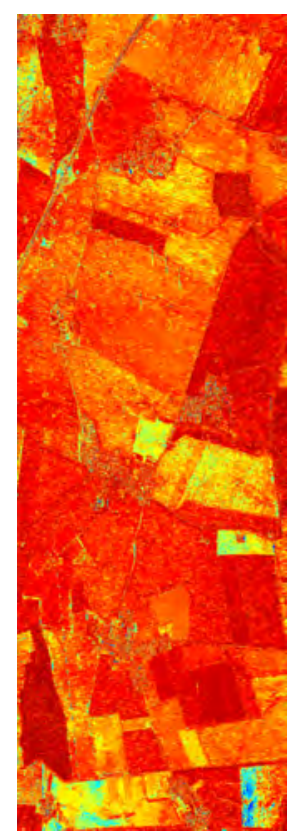

(c) $H$ 5th July

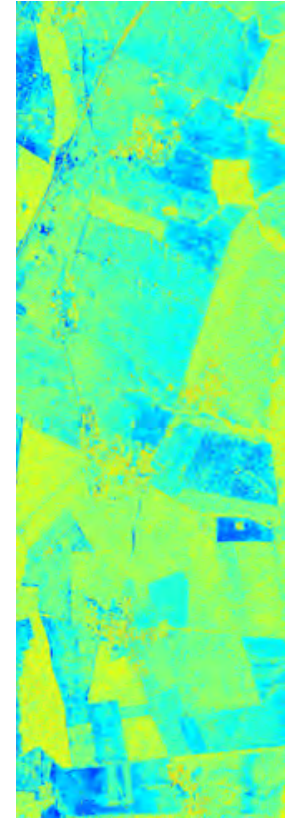

(f) $\bar{\alpha} 5$ th July
Fig. 6. Entropy $(H)$ and averaged alpha angle $(\bar{\alpha})$ eigendecomposition parameters for the three selected acquisitions.

the correlation between the areas affected by large changes obvious. In fact, since the change measures in Figs. 5a and 10 do not distinguish between increase or decrease, they may correspond to an increase $(\lambda>0 \mathrm{~dB})$ or a decrease $(\lambda<0 \mathrm{~dB})$ of the backscattered power and appear reddish in Fig. 7a or bluish in Fig. 7c, respectively.

Fig. 9 shows the histograms of the generalized eigenvalues for both dates, expressing the overall behavior of the polarimetric change. As it may be seen in Fig. 9a, there is a wide 


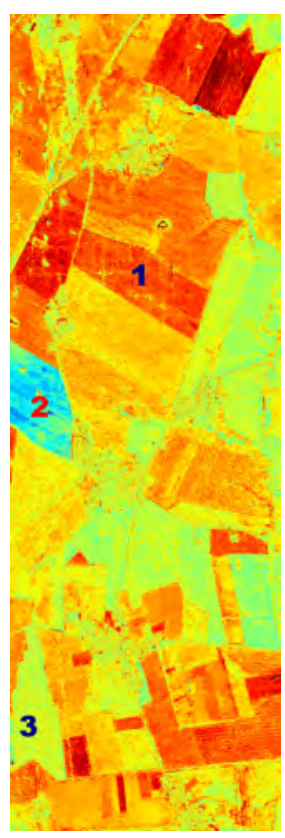

(a) $\lambda_{1}$

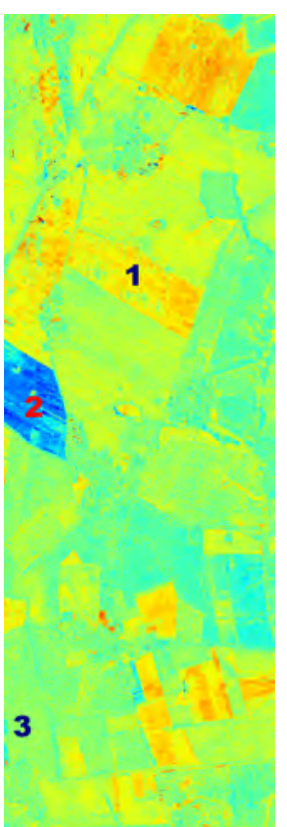

(b) $\lambda_{2}$

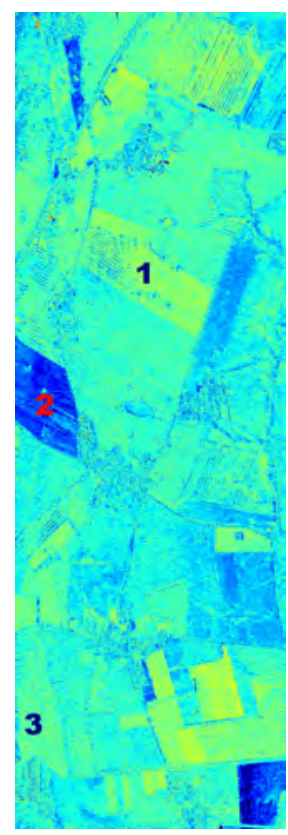

(c) $\lambda_{3}$

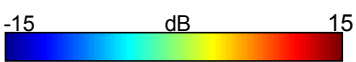

Fig. 7. Magnitude representation of the three generalized eigenvalues (in $\mathrm{dB}$ ) depicting the change between the acquisitions from 19th April to 7 th June.

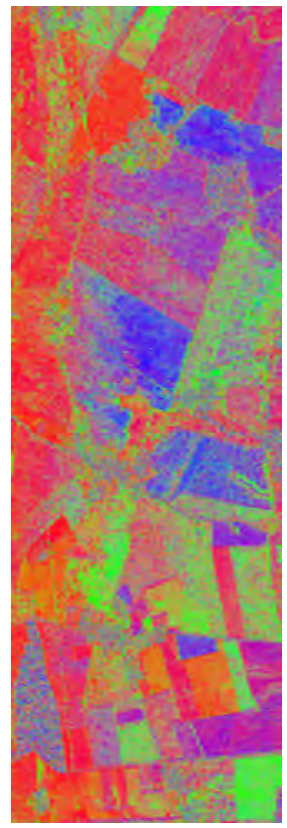

(a) $\mathbf{w}_{1}$

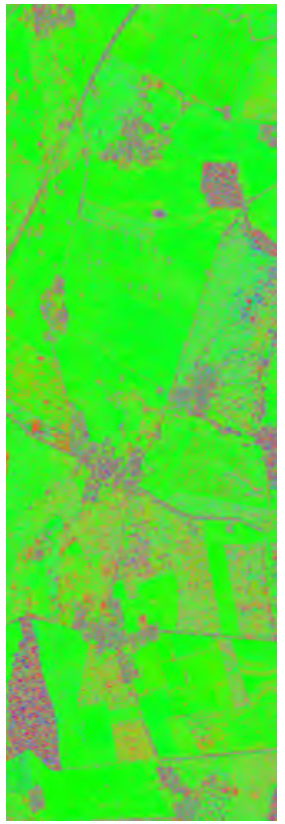

(b) $\mathbf{w}_{2}$

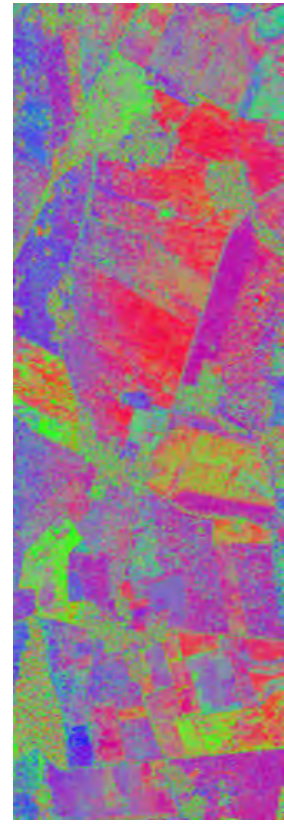

(c) $\mathbf{w}_{3}$
Fig. 8. Pauli representation of the three generalized eigenvectors depicting the change between the acquisitions from 19th April to 7th June.

range of changes occurring between the 19th April and 7th June. The peak of $\lambda_{1}$ is at about $6 \mathrm{~dB}$, while the peak of $\lambda_{3}$ is at $-2.5 \mathrm{~dB}$, indicating a relevant increase as well as decrease of the power of individual scattering contributions. However, most of the $\lambda_{i}$ values are larger than $0 \mathrm{~dB}$, indicating that the increasing changes are dominating. The changes occurring in

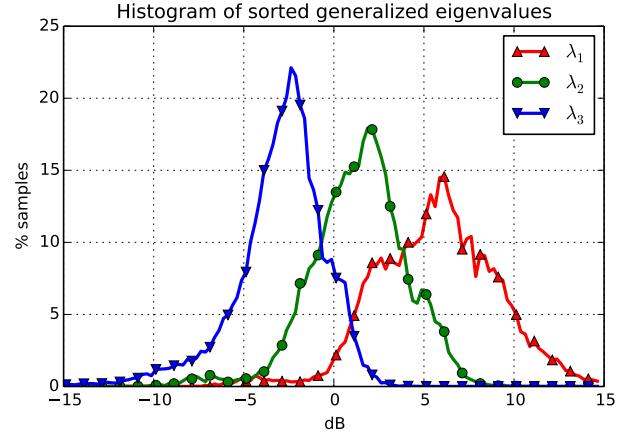

(a) 19th April to 7 th June

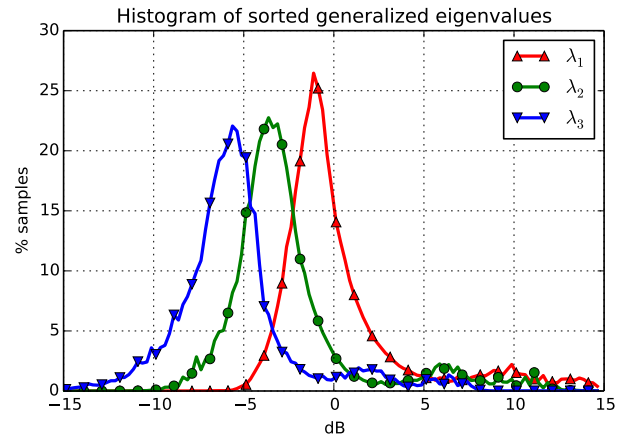

(b) 7th June to 5th July

Fig. 9. Histograms of the generalized eigenvalues $\lambda_{i}$ (a) from 19th April to 7 th June and (b) from 7th June to 5th July.

the time between the 7 th June and 5th July have a different behavior as shown in Fig. 9b. The peaks of all the histograms are negative, indicating a decrease in power of the scattering contributions for most of the areas. Moreover, the shape of the histograms is also different, with narrower distributions in Fig. 9b than on Fig. 9a.

It is important to note here that the full change information is expressed by the full set of generalized eigenvalues (shown in Fig. 7) and eigenvectors (shown in Fig. 8). For example, the wheat field marked with " 1 " in Fig. 7 is characterized by a strong increase in $\lambda_{1}$ (Fig. 7a) associated to a change in $\mathbf{w}_{1}$ (shown in Fig. 8a) but no significant change in $\lambda_{3}$ (Fig. 7c). In contrary, the maize field marked with " 2 " in Fig. 7 , shows the strongest change (a decrease) in $\lambda_{3}$ associated to the polarization state $\mathbf{w}_{3}$. Fields that do not change significantly are characterized by generalized eigenvalues close to $0 \mathrm{~dB}$ and appear in green in all $\lambda_{i}$ images. Such a case is the forested area marked with " 3 " in Fig. 7. For these no-change areas, the obtained generalized eigenvectors (see Fig. 8) are noisier corresponding to variations induced by speckle. This is in accordance with the results discussed in [40]. In conclusion, the change interpretation might only be achieved by combining the information provided by the generalized eigenvalues and eigenvectors shown in Figs. 7 and 8.

In order to consider all this information, the change representation proposed in Section III-C weights the contribution of each generalized eigenvector $\mathbf{w}_{i}$ according to the magnitude of the change given by the corresponding generalized eigenvector 


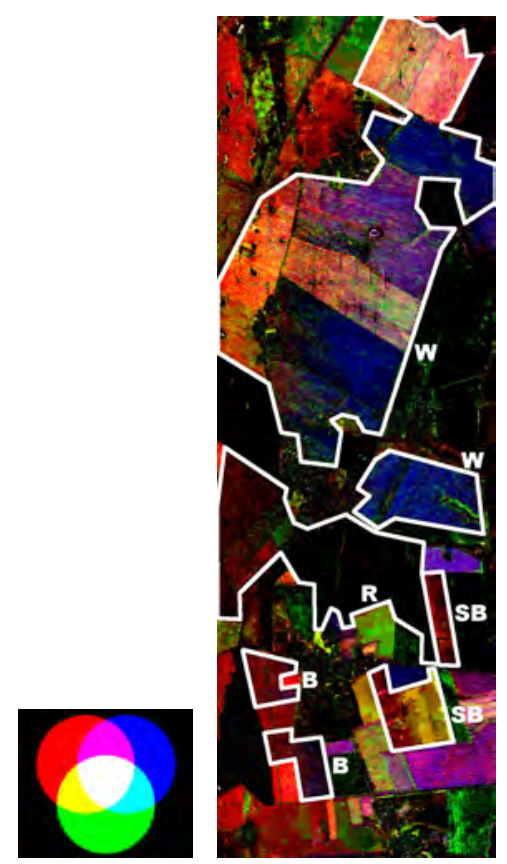

(a) $\mathbf{p}_{\text {inc }}$

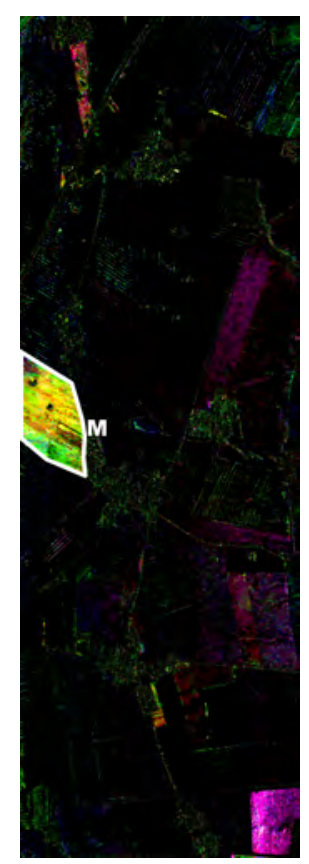

(b) $\mathbf{p}_{\text {dec }}$
Fig. 10. Polarimetric change representation for differences from 19th April to 7th June acquisitions. The labels represent the crop type: (W) wheat, (B) Barley, (R) rape, (M) maize and (SB) sugar beet.

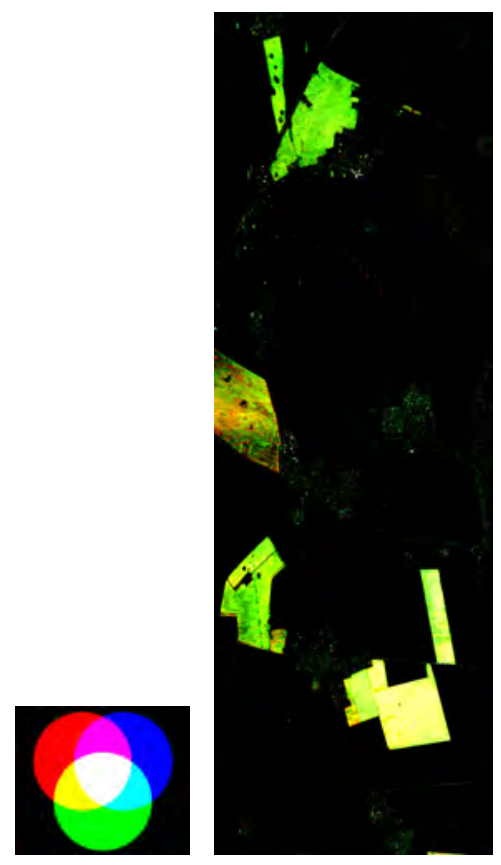

(a) $\mathbf{p}_{\text {inc }}$

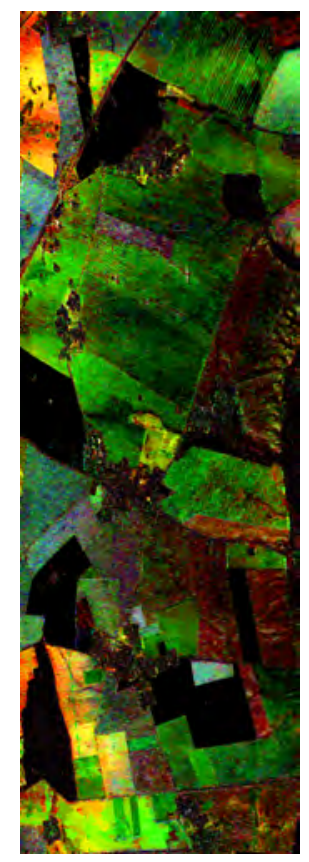

(b) $\mathbf{p}_{\text {dec }}$
Fig. 11. Polarimetric change representation for differences from 7th June to 5 th July acquisitions.

$\lambda_{i}$. This representation combines the (increasing and decreasing) change information for all the $\lambda_{i}$ and $\mathbf{w}_{i}$ into two images. Figs. 10 and 11 show the change representation proposed in (17) and (18), i.e. the Pauli representation of the weighted polarization states on which the changes are observed. Both RGB images are scaled from 3 to $10 \mathrm{~dB}$. This scaling filters out the small changes introduced by speckle as all areas with an intensity change below $\pm 3 \mathrm{~dB}$ appear in black.

Fig. 12 shows photographs of the relevant crop fields acquired at the three intensive campaign days. The numbers correspond to the field numbers used in the land-use map in Fig. 2. The changes relevant for the interpretation of Fig. 10 are between the 1st and 2nd row of Fig. 12. Similarly, the changes appearing in Fig. 11 correspond to the differences between the 2nd and 3rd row of Fig. 12.

Looking on Fig. 10, a large number of fields appear in the $\mathbf{p}_{i n c}$ image, indicating an increase, mostly in red and blue colors. According to the ground measurements in Fig. 3, this is mainly due to plant growing, as depicted in the vegetation height plot in Fig. 3a. Specially for wheat, barley and rape a clear increase in vegetation height is given for most of the fields where the increase is detected in Fig. 10a. For sugar beet and maize crops a smaller increase in vegetation height is observed as they are planted later. This is also clearly indicated by the BBCH phenology plot on Fig. 4. The changes on the sugar beet and winter barley fields, that appear in red and yellow colors in Fig. 10a are shown in Figs. 12f-121 and 12c$12 \mathrm{i}$, respectively.

The rape fields do not follow this trend, even if they are characterized by an significant increase of height as indicated by Fig. 3a and shown in Figs. 12d-12j. Only for a single field located in near-range, i.e. at the left part of the change image in Fig. 10a, an increase is visible. All other rape fields appear black indicating that the polarimetric change is below $\pm 3 \mathrm{~dB}$. The reason for this is probably in the dominant volume scattering contribution that characterizes the rape fields in both acquisitions: the plant density, the wet biomass and the water content (see Fig. 3b) is larger for the rape than for other types of crops in the scene. This increases volume scattering and limits at the same time the penetration of the radar waves through the vegetation to the ground, preventing that the change in vegetation height is translated into a change in the polarimetric response.

For the winter wheat fields, a strong increase is detected in Fig. 10a, associated to the plant growth, as shown in the plot in Fig. 3a and in Figs. 12a-12g and 12b-12h. However, it is remarkable that some of the fields appear reddish while others bluish, although no clear difference is observed between these fields. The different colors indicate that the change occur at different polarizations, i.e. affects different scattering contributions, which may be induced by the fact that these fields are in a different phenological state. Unfortunately, the $\mathrm{BBCH}$ measurements in Fig. 4 represent only the average crop type evolution and are not specific for each field. Moreover, there are missing measurements for the wheat crop at these dates. Fortunately, during the intensive measurement campaigns a large amount of photographs were collected, that allows to visualize these differences. Some photographs of the wheat plants on the distinct fields are shown in Fig. 13. They make clear that the fields appearing in red in Fig. 10a have not started with the heading process (Figs. 13a-13c), whereas the fields appearing in blue color have started this process (Figs. 13d13f).

Note that this does not mean that the method is able to detect 


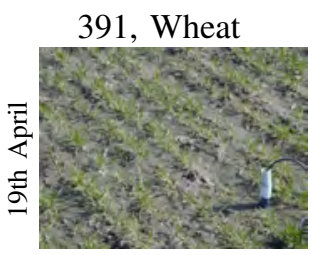

(a)

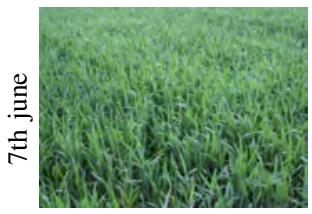

(g)

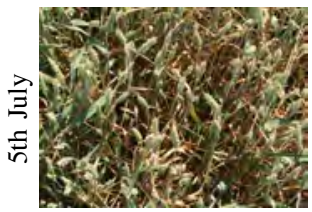

(m)
250, Wheat

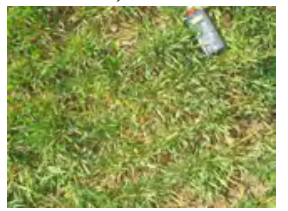

(b)

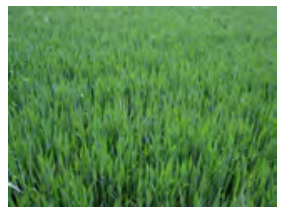

(h)

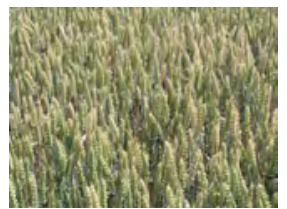

(n)

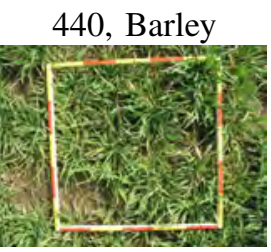

(c)

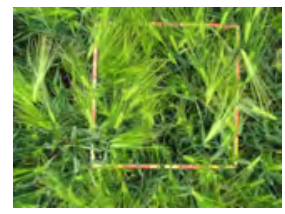

(i)

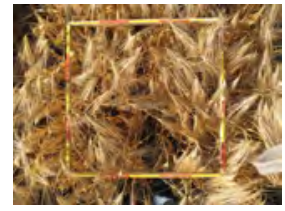

(o)

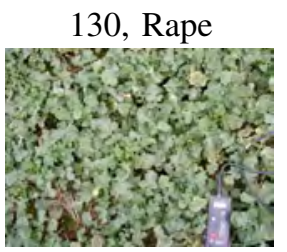

(d)

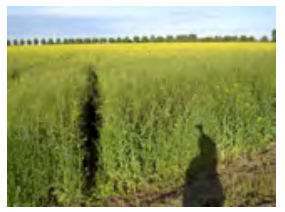

(j)

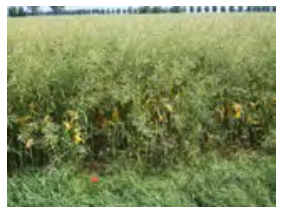

(p)
222, Maize

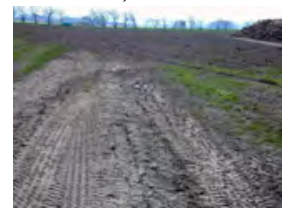

(e)

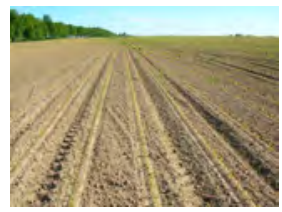

(k)

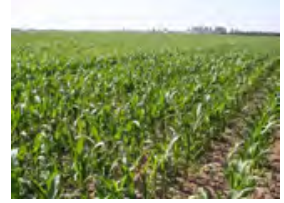

(q)
102, Sugar beet

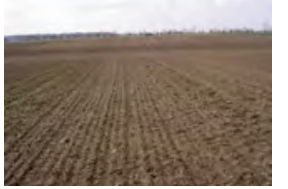

(f)

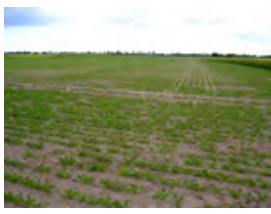

(1)

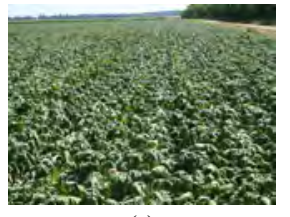

(r)

Fig. 12. Photographs of the different fields discussed. The field number corresponds to the land-use map in Fig. 2. The columns represent different fields, as indicated on top, while the rows indicate the three intensive measurement dates, as indicated in the left.

the heading process of the plant directly. It rather means that the fields have a different polarimetric change between this two acquisitions, probably due to the different phenological evolution which alters their polarimetric response in both acquisitions.

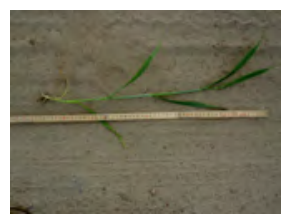

(a) Field 391

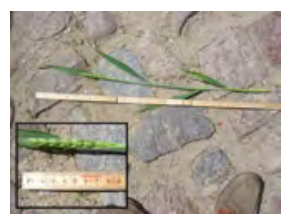

(d) Field 310

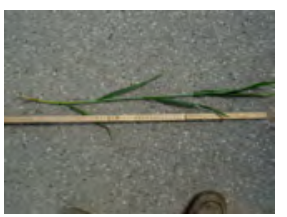

(b) Field 291

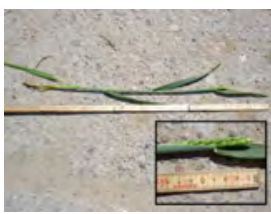

(e) Field 292

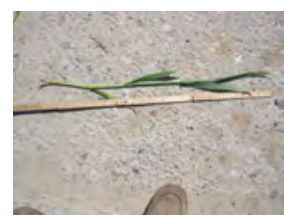

(c) Field 221

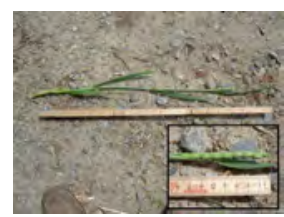

(f) Field 250
Fig. 13. Photographs of the different winter wheat plants on 7 th June. A zoom is shown in the black rectangle for those plants that have started the heading process in (d)-(f).

Fig. 11 shows the polarimetric change maps associated to the changes occurring between the 7th of June and the 5th of July. Compared to the change maps associated to the changes occurring between the 19th of April and the 7th of June (see Fig. 10) they reflect a different change behavior, dominated by a intensity decrease for most of the fields. The ground measurement in Fig. 3 indicate that two types of change are ongoing between these dates. For maize and sugar beet an increase in vegetation height is observed, while the plant water content remains constant. Note that these crops grow later, compared to the other crops, as it may be seen in Fig. 4. The discussed changes may also be observed in the photographs in Fig 12. The growth is also documented by $12 \mathrm{k}-12 \mathrm{q}$ for the maize and Figs. 121-12r for the sugar beet. Accordingly these fields appear as increasing intensity changes in green (and some red) colors in Fig. 11a. For all other crops in the scene (i.e. winter wheat, rape, winter barley), Fig. 3 indicates that the vegetation height remains constant but the water content decreases. These crops are at the time of the second acquisition (7th of June) already grown up and then, in the time between the second and the third (5th of July) acquisition they undergo the plant maturation and the drying out at the end of the phenological stage. Especially the plant drying is associated to a decrease in the backscattered power clearly visible in the photographs in Fig 12, indicated by the yellowish color for the winter wheat (Figs. 12g-12m), rape (Figs. 12j-12p), and specially winter barley (on Figs. 12i-12o). However for the different crops the changes imposed by the plant drying appear in $\mathbf{p}_{\text {dec }}$ (Fig. 11b) with different colors, i.e., are associated to different scattering mechanisms. The winter wheat fields appear in green (indicating a decrease of volume scattering), whereas a more yellowish color is obtained for the winter barley crops (i.e. decrease of volume and dihedral). In the case of the rape, the color depends on the incidence angle: the rape fields in the left part of the image (in near-range) appear more greenish (decrease of volume scattering). This may be because of the larger penetration associated to the steeper incidence angles. Note that these are the same fields where the plant growth was detected in Fig. 10a. The rape fields on the right part of the map (i.e. in far-range) appear reddish (indicating decrease of dihedral scattering).

Finally, it is important to mention that most of the discussed changes appearing in the change representation images shown in Figs. 10 and 11, may also be detected in the Pauli RGB images and the entropy and mean Alpha angle maps, shown in Figs. 1 and 6, respectively. However, their identification and interpretation is either more vague and unclear or not always 
possible.

The polarimetric change representation for the acquisitions from the 19th of April to the 5th of July will not be discussed in this section. However, it can be inferred from the previous two analysis. From a mathematical point of view, the polarimetric change analysis will result in the multiplicative combination of the previous two, as previously indicated in (15). Alternatively, the next section describes a systematic method for change analysis in time-series.

\section{B. Results over all the acquisitions}

The AgriSAR 2006 campaign comprises a time-series of 12 L-band fully polarimetric acquisitions at the same geometry, from the 12th of May to the 4th of August. This allows 66 combinations of different acquisitions pairs for change analysis. The previous approach, then, becomes infeasible for the individual analysis of the $\mathbf{p}_{i n c}$ and $\mathbf{p}_{d e c}$ for each of those pairs. Note that, although the different acquisition pairs are related according to (16), the full picture of all the change pairs may give a valuable idea of how successive changes combine with each other. Therefore, for the analysis of the changes among all the acquisitions of the data set a different approach is required. A change matrix representation is proposed, which contains the results of the previously mentioned change representation between all the acquisition pairs [41]. Instead of comparing the results pixel by pixel, a set of regions is defined over the image, corresponding to homogeneous areas of the agricultural fields, and the change analysis is performed over the average covariance matrices in these regions at different dates. The horizontal and vertical axes of the change matrix correspond to the acquisition dates of each image. For each combination of acquisition pairs $(i, j)$, in the upper diagonal the $\mathbf{p}_{i n c}$ representation between the $i$ and $j$ acquisitions is shown, whereas in the lower diagonal $(j, i)$ the $\mathbf{p}_{d e c}$ between $i$ and $j$ is displayed.

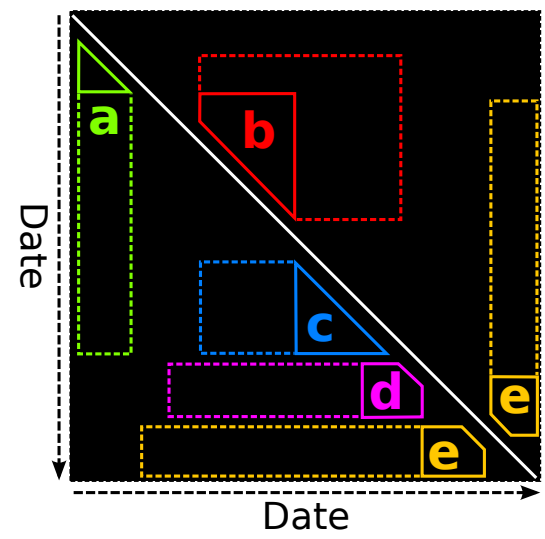

Fig. 14. Scheme of the change matrix and the main expected detected changes. (a) Seedbed preparation and planting, (b) plant growing, (c) fruit development, (d) plant drying and (e) harvest.

Fig. 14 presents a scheme of the change matrix and depicts the main phenological and/or development change signatures for agricultural crops that may be detected in different colors, according to the results already analyzed in Section IV-A: a Seedbed preparation and planting (e.g. Figs. 12e to 12k). In this case a decrease in the surface roughness due to the seedbed preparation is translated into a decrease of the backscattered signal, appearing in the lower diagonal of the change matrix.

b The plant growing process (e.g. Figs. 12a to $12 \mathrm{~g}$ ), corresponding to phenological stages 30-59 in the $\mathrm{BBCH}$ scale, including the stem elongation, booting and heading. When the plant grows, an increase of the radar backscattering signal is obtained due to the increased reflectivity of the plant. In this case, then, it appears in the upper diagonal of the change matrix.

c The fruit development (e.g. Figs. $12 \mathrm{~g}$ to $12 \mathrm{~m}$ ), corresponding to phenological stages 60-79 in the $\mathrm{BBCH}$ scale, related to the flowering and fruit development of the plant. After the growing process, the plant develops its fruit and a moderate reduction in water content is produced, which is perceived as a decrease in the $\mathbf{p}_{d e c}$ change representation.

d The plant drying (e.g. Figs. 12i to 12o), corresponding to the $\mathrm{BBCH}$ scale stages $80-99$, including ripening and senescence. In the final stages of the plant before harvesting, a strong decrease in water content is observed, which is translated into a strong decrease of the backscattering.

e The crop harvesting, associated with a strong decrease of the volume scattering due to the removal of most of the plant. However, an additional increase may also be seen in different polarization states, representing the increase of the ground scattering component. In some cases, the lower part of the plant is left over the field after harvesting, contributing also to this increase of the ground scattering. Accordingly, harvesting may be mapped in the lower but also in the upper diagonal block of the change matrix.

For the interpretation of the change matrix representation it is important to keep in mind that the amount and nature of the change processes represented at each entry also depend on the temporal distance between the associated acquisitions. In general, for temporally adjacent acquisitions only one type of change occurs, assuming a sufficiently high temporal sampling of the ongoing change process. These cases are indicated in Fig. 14 by the matrix areas framed by solid lines. The same change process may be visible for a longer time interval and so present at multiple image pairs, as indicated in Fig. 14 by the matrix areas framed by dashed lines. For instance, the red dashed area covers the combination of the plant growing process with the previous seedbed preparation and the subsequent plant drying process. In these areas, the obtained change representation will depend on how the combination of these physical changes are perceived by the sensor, according to the multiplicative combination of the different $\mathbf{M}$ matrices as described in (16). Then, the obtained results in these areas will depend on the crop type, the plant geometry and density, the radar penetration, etc.

In the case of the AgriSAR 2006 campaign, all changes described in Fig. 14 will not be visible for each crop type as 
the campaign does not cover the whole phenological cycle of all crops, as it may be seen in Fig. 4. However, the temporal shift between the phenological cycle of the different crops, caused by the fact that some crops are planted before others, allows the detection of each change for at least one crop type.

Fig. 15 shows the change matrices for six fields: two wheat fields, a barley, a rape, a maize and a sugar beet field. The observed changes from those described in Fig. 14 have been labeled employing the same colors for an easy identification in all the change matrices. For wheat, the two fields selected correspond to the two different changing behaviors appearing in Fig. 10a. In Fig. 15, the scaling of the $\mathbf{p}_{i n c}$ and $\mathbf{p}_{d e c}$ on the change matrices is between 1 and $8 \mathrm{~dB}$.

As it may be seen, the two different wheat growing changes observed in Fig. 10a may also be seen in Figs. 15a and 15b, especially on the first row, which correspond to the comparison of the first acquisition, on the 19th of April, with the rest of acquisitions, as the change analyzed in Section IV-A. Apart from these small color changes, the change matrices for the two wheat fields are very similar, having the same change regions associated to the growing period, the fruit maturation and the plant drying as labeled in Figs. 15a and 15b. Comparing Figs. 15a to $15 \mathrm{c}$, it may be observed that the wheat and barley crops present similar growing periods, but the drying process (marked in blue) is faster for the barley, as seen in Fig. 15c. For the barley even the harvesting is observed at the end of the acquisition campaign, marked in yellow color. Interestingly, the harvesting phase appears as a combination of a decrease of volume scattering due to the elimination of the plant structure and an increase of the ground component. Therefore, the decrease and increase observed due to the harvest appear with different colors, indicating a different type of change.

In the rape field, as discussed before in Section IV-A, the plant growth is not observed due to the incapacity of the radar to penetrate to the ground. However, the later changes in the vegetation due to the fruit maturation and plant drying clearly appear in the change matrix as marked in Fig. 15d. For the maize and sugar beet crops, shown in Figs. 15e and 15f which are planted later, only the growing period is observed, marked in a red polygon. Additionally, in the maize field even the seedbed preparation and planting may be seen in the first acquisitions, labeled in green color.

It is worth noticing that for most of the cereal crops, including wheat, barley and maize, the change corresponding to the growing period, labeled in red, starts around the stem elongation phase, equivalent to $30 \mathrm{~s}$ in the $\mathrm{BBCH}$ scale, depicted in Fig. 4. However, for the sugar beet crop this change is detected much earlier, in the leaf development phase, which corresponds to $10 \mathrm{~s}$ in the $\mathrm{BBCH}$ scale. This could be produced by the fact that the sugar beet leaves are bigger, having a higher surface than those of the cereals, or also due to the higher water content of these crops. In any case, this exemplifies how the changes and evolution of the different crop types are perceived unevenly by the radar.

Looking at the different change matrices presented in Fig. 15, it may be seen that they are distinct for every type of crop, as every crop follows a different temporal evolution.
Even the two distinct changes for the wheat fields, shown in Figs. $15 \mathrm{a}$ and $15 \mathrm{~b}$, are very similar between them. Then, the change matrix may be seen as a crop signature, which may be very useful for crop identification and land use classification.

Apart from the big generic change processes labeled according to the scheme in Fig. 14, some small changes or dots may also be observed. This is especially the case for the acquisition of the 11th of May, since smaller changes may be observed in this date for some of the change matrices. This was a particularly dry acquisition as no rain was measured during the two weeks before. Hence, a faint spot is observed as a decrease in the change matrix, especially for the species with lower vegetation height, as wheat, barley and sugar beet. However, since this change is an isolated fact occurring only in one acquisition, the spot observed in the change matrices is not continued in time, as opposite to the other changes observed due to the crop evolution and marked in the different colors. Therefore, sufficiently dense time series are desired in order to be able to separate these isolated cases from the rest of the temporal evolution.

\section{CONCLUSiOnS}

A new change detection technique able to quantify and interpret changes in multi-temporal polarimetric SAR acquisitions has been proposed. It is based on the generalized eigendecomposition of the covariance matrices of the individual acquisitions. The generalized eigenvalues are related to the polarimetric contrast ratio, and thus to the maximum and minimum changes between the covariance matrices. The generalized eigenvectors represent the polarization states (i.e. scattering mechanisms) associated to these changes. This allows not only the estimation of the change magnitude but also the physical interpretation of the occurred changes. Weighting the scattering mechanisms by the amount of their change allows the visualization and interpretation of the change information in terms of two Pauli change images that comprise the type of change in the color information and the magnitude of change in the intensity.

The proposed technique has been employed and tested for monitoring the phenological development of agricultural vegetation on the multi-temporal AGRISAR 2006 L-band campaign data. The analysis focused on 5 important crop types: wheat, barley, rape, maize and sugar beet. For the different crop types, the proposed technique was able to detect and interpret a number of changes related to crop development as the growing, maturation and drying processes. Even for the same crop type, for example the winter wheat crops, a different change behavior can be observed at different fields. This is related to a temporal shift of the phenological cycle at the different fields, as on some fields the heading process started earlier than on others.

For a better visualization and interpretation of the changes occurring in long time series, the change matrix representation has been introduced. It has proven to be able to indicate and interpret changes related to important development stages and/or transitions between them, including the seedbed preparation, planting, growing, fruit development, senescence and 


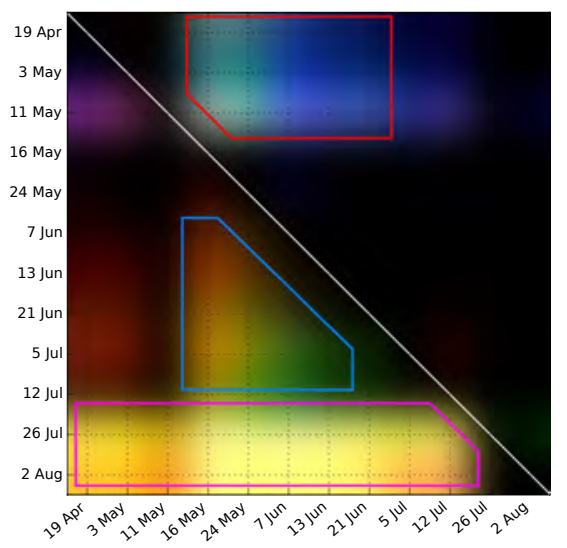

(a) Wheat, field 292

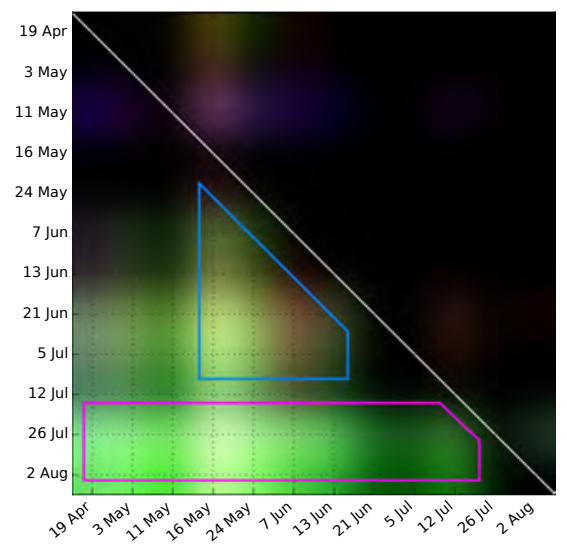

(d) Rape, field 101

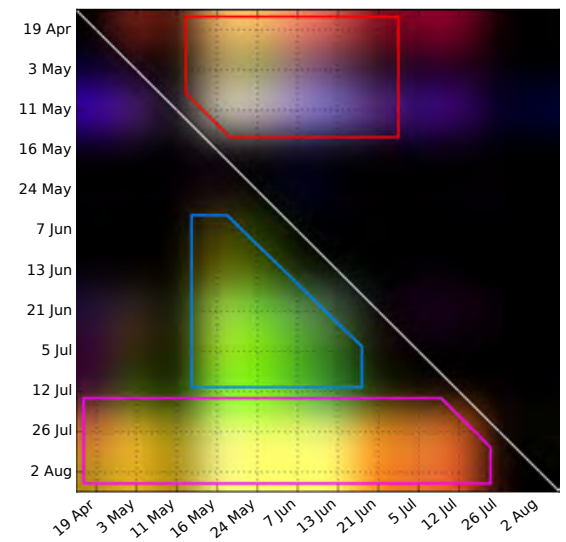

(b) Wheat, field 391

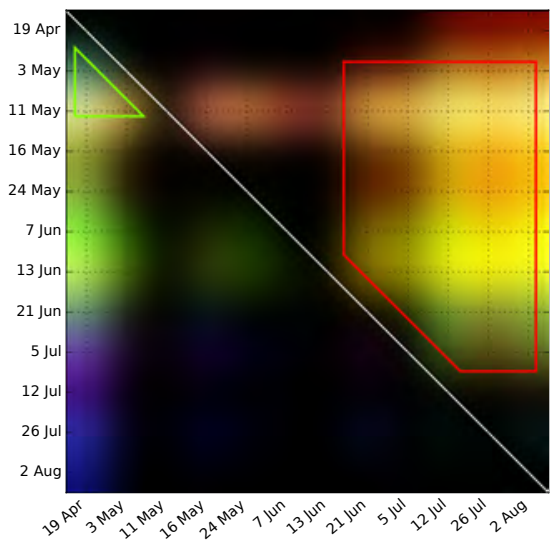

(e) Maize, field 222

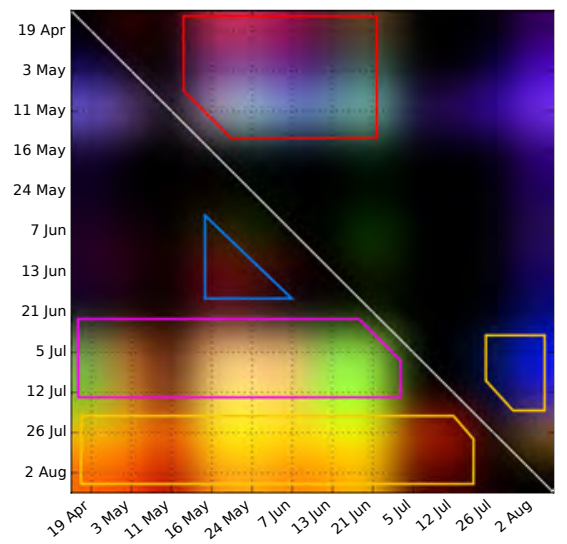

(c) Barley, field 440

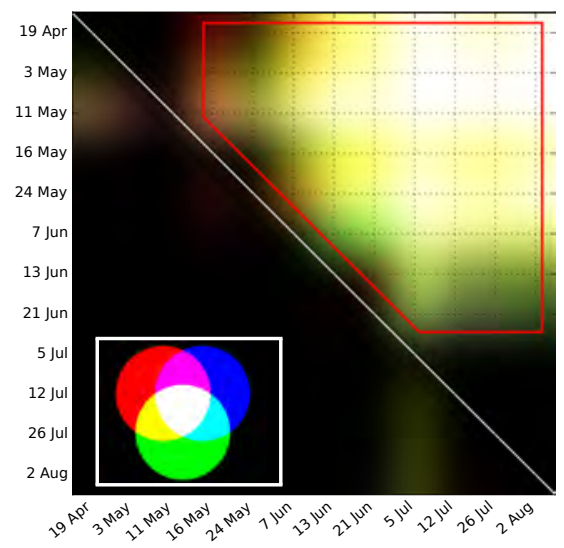

(f) Sugar beet, field 460

Fig. 15. Change matrices for different types of crops. The upper diagonal represents the increase $\mathbf{p}_{\text {inc }}$ and the lower diagonal the decrease $\mathbf{p}_{\text {dec }}$ between each acquisition pair. The visible changes of those described in Fig. 14 are marked, employing the same colors.

harvest. The results indicate the potential of this approach for monitoring the temporal evolution of agricultural plants. It is worth mentioning that the interpretation of the change matrices is strongly dependent on the frequency at which the SAR acquisitions are made and it may be different for other frequencies.

Finally, it is worth to mention that the proposed polarimetric change analysis uses only a subset of the parameters provided by the generalized eigendecomposition, namely the intensities of the changing polarization states. The associated phase information remains unused and may be further exploited in the future.

\section{ACKNOWLEDGMENT}

The work presented in this paper has been conducted under the funding of the HGF Alliance HA-310 'Remote Sensing and Earth System Dynamics'.

\section{REFERENCES}

[1] W. M. Brown, "Synthetic aperture radar," IEEE Transactions on Aerospace and Electronic Systems, vol. AES-3, no. 2, pp. 217-229, March 1967.

[2] J. A. Ogilvy and H. M. Merklinger, "Theory of wave scattering from random rough surfaces," The Journal of the Acoustical Society of America, vol. 90, no. 6, pp. 3382-3382, 1991. [Online]. Available: https://doi.org/10.1121/1.401410

[3] J. Lee, "Refined filtering of image noise using local statistics," Computer graphics and image processing, vol. 15, no. 4, pp. 380-389, 1981.

[4] G. Vasile, E. Trouve, J.-S. Lee, and V. Buzuloiu, "Intensity-driven adaptive-neighborhood technique for polarimetric and interferometric SAR parameters estimation," Geoscience and Remote Sensing, IEEE Transactions on, vol. 44, no. 6, pp. 1609-1621, 2006.

[5] A. Alonso-Gonzalez, C. Lopez-Martinez, and P. Salembier, "Filtering and Segmentation of Polarimetric SAR Data Based on Binary Partition Trees," Geoscience and Remote Sensing, IEEE Transactions on, vol. 50, no. 2, pp. 593-605, Feb 2012.

[6] C.-A. Deledalle, L. Denis, and F. Tupin, "Iterative Weighted Maximum Likelihood Denoising With Probabilistic Patch-Based Weights," Image Processing, IEEE Transactions on, vol. 18, no. 12, pp. 2661-2672, Dec 2009.

[7] J. J. Van Zyl, H. A. Zebker, and C. Elachi, "Imaging radar polarization signatures: Theory and observation," Radio science, vol. 22, no. 04, pp. 529-543, 1987. 
[8] S. Cloude, Polarisation: applications in remote sensing. Oxford University Press, USA, 2009.

[9] J. Lee and E. Pottier, Polarimetric radar imaging: from basics to applications. CRC Press, 2009.

[10] C. Elachi and J. van Zyl, Introduction To The Physics and Techniques of Remote Sensing, ser. Wiley Series in Remote Sensing and Image Processing. John Wiley \& Sons, 2006.

[11] F. Ulaby and C. Elachi, Radar polarimetry for geoscience applications, ser. Artech House remote sensing library. Artech House, 1990.

[12] J. Huynen, "Measurement of the target scattering matrix," Proceedings of the IEEE, vol. 53, no. 8, pp. 936-946, 1965.

[13] E. Krogager, "New decomposition of the radar target scattering matrix," Electronics Letters, vol. 26, no. 18, pp. 1525-1527, 1990.

[14] W. Cameron and L. Leung, "Feature motivated polarization scattering matrix decomposition," in Radar Conference, 1990., Record of the IEEE 1990 International. IEEE, 1990, pp. 549-557.

[15] A. Freeman and S. Durden, "A three-component scattering model for polarimetric SAR data," Geoscience and Remote Sensing, IEEE Transactions on, vol. 36, no. 3, pp. 963-973, 1998.

[16] R. Touzi, "Target scattering decomposition of one-look and multilook SAR data using a new coherent scattering model: The TSVM," in Geoscience and Remote Sensing Symposium, 2004. IGARSS'04. Proceedings. 2004 IEEE International, vol. 4. IEEE, 2004, pp. 24912494.

[17] S. Cloude and E. Pottier, "An entropy based classification scheme for land applications of polarimetric SAR," Geoscience and Remote Sensing, IEEE Transactions on, vol. 35, no. 1, pp. 68-78, 1997.

[18] K. Conradsen, A. Nielsen, J. Schou, and H. Skriver, "A test statistic in the complex Wishart distribution and its application to change detection in polarimetric SAR data," Geoscience and Remote Sensing, IEEE Transactions on, vol. 41, no. 1, pp. 4-19, Jan 2003

[19] A. Marino, S. Cloude, and J. Lopez-Sanchez, "A New Polarimetric Change Detector in Radar Imagery," Geoscience and Remote Sensing, IEEE Transactions on, vol. 51, no. 5, pp. 2986-3000, May 2013.

[20] A. Alonso-Gonzalez, C. Lopez-Martinez, and P. Salembier, "PolSAR Time Series Processing With Binary Partition Trees," Geoscience and Remote Sensing, IEEE Transactions on, vol. 52, no. 6, pp. 3553-3567, June 2014.

[21] C. A. Balanis, Advanced engineering electromagnetics. John Wiley \& Sons, 1999.

[22] S. R. Cloude and E. Pottier, "A review of target decomposition theorems in radar polarimetry," IEEE Transactions on Geoscience and Remote Sensing, vol. 34, no. 2, pp. 498-518, March 1996.

[23] B. V. Gnedenko and A. N. Kolmogorov, Limit distributions for sums of independent random variables, revised ed. Addison-Wesley, 1968.

[24] J. Goodman, "Some fundamental properties of speckle," JOSA, vol. 66, no. 11, pp. 1145-1150, 1976.

[25] N. Goodman, "Statistical analysis based on a certain multivariate complex Gaussian distribution (an introduction)," The Annals of Mathematical Statistics, vol. 34, no. 1, pp. 152-177, 1963.

[26] F. Barbaresco, "Interactions between Symmetric Cone and Information Geometries: Bruhat-Tits and Siegel Spaces Models for High Resolution Autoregressive Doppler Imagery," in Emerging Trends in Visual Computing, ser. Lecture Notes in Computer Science, F. Nielsen, Ed. Springer Berlin / Heidelberg, 2009, vol. 5416, pp. 124-163.

[27] A. Kostinski and W.-M. Boerner, "On the polarimetric contrast optimization," Antennas and Propagation, IEEE Transactions on, vol. 35, no. 8, pp. 988-991, Aug 1987.

[28] A. Swartz, H. Yueh, J. Kong, L. Novak, and R. Shin, "Optimal polarizations for achieving maximum contrast in radar images," Journal of Geophysical Research: Solid Earth, vol. 93, no. B12, pp. 15252 $15260,1988$.

[29] B. Parlett, The Symmetric Eigenvalue Problem. Society for Industrial and Applied Mathematics, 1998.

[30] Z. Bai, J. Demmel, J. Dongarra, A. Ruhe, and H. Van Der Vorst, Templates for the Solution of Algebraic Eigenvalue Problems: A Practical Guide, ser. Software, Environments and Tools. Society for Industrial and Applied Mathematics, 2000, ch. Generalized Hermitian Eigenvalue Problems.

[31] A. Marino and I. Hajnsek, "A Change Detector Based on an Optimization With Polarimetric SAR Imagery," Geoscience and Remote Sensing, IEEE Transactions on, vol. 52, no. 8, pp. 4781-4798, Aug 2014.

[32] S. R. Cloude, "Uniqueness of target decomposition theorems in radar polarimetry," in Direct and Inverse Methods in Radar Polarimetry: Part 1, W.-M. Boerner, Ed. Dordrecht: Springer Netherlands, 1992, pp. $267-296$.
[33] R. A. Horn and C. R. Johnson, Matrix analysis. Cambridge university press, 2012.

[34] A. Alonso-Gonzalez, T. Jagdhuber, and I. Hajnsek, "Exploitation of agricultural polarimetric sar time series with binary partition trees," in International Workshop on Science and Applications of SAR Polarimetry and Polarimetric Interferometry, POLinSAR, vol. 729, 2015.

[35] A. Alonso-González, T. Jagdhuber, and I. Hajnsek, "Agricultural monitoring with polarimetric sar time series," in 2015 8th International Workshop on the Analysis of Multitemporal Remote Sensing Images (Multi-Temp). IEEE, 2015, pp. 1-4.

[36] A. Marino and A. Alonso-González, "An optimization of the difference of covariance matrices for polsar change detection," in 2017 IEEE International Geoscience and Remote Sensing Symposium (IGARSS). IEEE, 2017, pp. 5315-5318.

[37] Microwaves German Aerospace Center and Radar Institute (HR), AGRISAR 2006 Agricultural Bio-/Geophysical Retrieval from Frequent Repeat SAR and Optical Imaging. European Space Agency, Paris, France, May 2008, Final Report.

[38] U. Meier, H. Bleiholder, E. Weber, P. Lancashire, C. Feller, L. Buhr, M. Hess, H. Wicke, H. Hack, R. Klose et al., "Growth stages of monoand dicotyledonous plants. BBCH Monograph," Federal Biological Research Centre for Agriculture and Forestry, 2001. [Online]. Available: http://pub.jki.bund.de/index.php/BBCH/article/viewFile/515/464

[39] J. M. Lopez-Sanchez, J. D. Ballester-Berman, and I. Hajnsek, "First Results of Rice Monitoring Practices in Spain by Means of Time Series of TerraSAR-X Dual-Pol Images," IEEE Journal of Selected Topics in Applied Earth Observations and Remote Sensing, vol. 4, no. 2, pp. 412 422, June 2011

[40] C. Lopez-Martinez, A. Alonso-Gonzalez, and X. Fabregas, "Perturbation Analysis of Eigenvector-Based Target Decomposition Theorems in Radar Polarimetry," Geoscience and Remote Sensing, IEEE Transactions on, vol. 52, no. 4, pp. 2081-2095, April 2014.

[41] A. Alonso-Gonzalez, H. Joerg, K. Papathanassiou, and I. Hajnsek, "Change analysis and interpretation in polarimetric time series over agricultural fields at C-band," in Proceedings of EUSAR 2016: 11th European Conference on Synthetic Aperture Radar. VDE, 2016, pp. $1-6$.

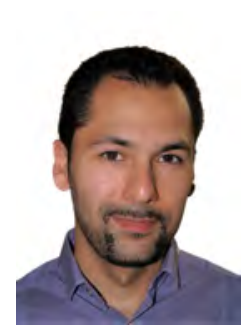

Alberto Alonso-González received the B.Sc. degree in computer science, the M.Sc. degree in telecommunication engineering and the Ph.D. degree from the Technical University of Catalonia (UPC), Barcelona, Spain, in 2007, 2009 and 2014 respectively.

From 2009 to 2014, he was with the Signal Theory and Communications Department (TSC), Technical University of Catalonia. Since June 2014 he is with the Microwaves and Radar Institute (HR) of the German Aerospace Center (DLR) as a member of the Polarimetric SAR Interferometry research group. His research interests include multidimensional synthetic aperture radar (SAR), SAR polarimetry and interferometry, digital signal and image processing. Currently, his main research activities are focused on SAR data modeling and decomposition techniques for the retrieval of biophysical information for agricultural and forest monitoring.

Dr. Alonso-González was the recipient of the First Place Student Paper Award at the EUSAR 2012 Conference. In 2016, he received the Extraordinary Doctoral Thesis Award, by the Technical University of Catalonia (UPC). 


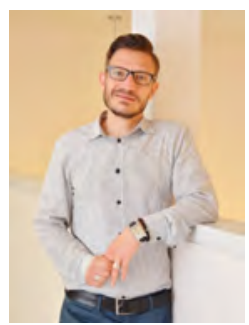

Carlos López-Martínez (S'97-M'04-SM'11) received the MSc. degree in electrical engineering and the Ph.D. degree from the Universitat Politècnica de Catalunya, Barcelona, Spain, in 1999 and 2003, respectively.

Dr. Lopez-Martinez is Associate Professor in the area of remote sensing and microwave technology in the Universitat Politècnica de Catalunya, Barcelona, Spain. He has a large professional international experience at DLR (Germany), at the University of Rennes 1 (France), and as a group leader of the Remote Sensing and Natural Resources Modelling team in the Luxembourg Institute of Science and Technology (Luxembourg). His research interests include Synthetic Aperture Radar (SAR) theory, statistics and applications, multidimensional SAR, radar polarimetry, physical parameter inversion, advanced digital signal processing, estimation theory, and harmonic analysis.

Dr. López-Martínez has authored more than 200 articles in journals, books, and conference proceedings, and received the EUSAR 2002 Conference Student Prize Paper Award, co-authored the paper awarded with the EUSAR 2012 Conference First Place Student Paper Award, and received the IEEEGRSS 2013 GOLD Early Career Award. Dr. López-Martínez has broad academic teaching experience from bachelor, master, and Ph.D. levels to advanced technical tutorials presented at international conferences and space and research institutions worldwide. $\mathrm{He}$ is an associate editor of the IEEEJSTARS journal and the MDPI Remote Sensing, acting also as invited guest editor for several special issues. He has collaborated in the Spanish PAZ and the ESA's SAOCOM-CS missions, in the proposal of the Parsifal mission and he is member of the ESA's Sentinel ROSE-L Mission Advisory Group. He was appointed vice-president of the IEEE-GRSS Spanish chapter, and in 2016 he became its secretary and treasurer. From 2011 Dr. LópezMartínez collaborates with the IEEE-GRSS Globalization initiative in Latin America, contributing to the creation of the IEEE-GRSS Chilean chapter and the organization of the 2020 LAGIRSS conference, being appointed as Latin America liaison in 2019. He is also co-chair of the Tutorial Technical Committee of the Indian 2020 InGARSS conference.

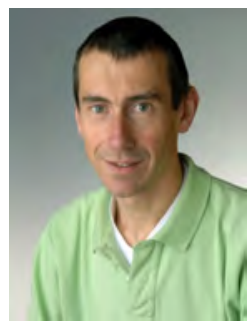

Konstantinos P. Papathanassiou (M'02, SM'03, FM'14) received the Dipl. Ing degree in 1994 and the Dr. degree in 1999 from the Technical University of Graz, Austria.

From 1992 to 1994 he was with the Institute for Digital Image Processing (DIBAG) of Joanneum Research, in Graz, Austria. Between 1995 and 1999 he worked at the Microwaves and Radar Institute (HR) of the German Aerospace Center (DLR), in Oberpfaffenhofen, Germany. From 1999 to 2000 he was an EU post-doctoral fellow with Applied Electromagnetics (AEL) in St. Andrews, Scotland. Since October 2000 he is again with the Microwaves and Radar Institute (HR) of the German Aerospace Center (DLR). Actually he is a senior scientist leading the Information Retrieval research group at DLR-HR. His main research interests are in polarimetric and interferometric processing and calibration techniques, polarimetric SAR interferometry, and the quantitative parameter estimation from SAR data as well as in SAR mission design and SAR mission performance analysis $\mathrm{He}$ is member of DLR's TanDEM-X and Tandem-L science teams, JAXA's ALOS-PalSAR Cal-Val teams, ESA's BIOMASS Mission Advisory Group and JAXA's Carbon and Kyoto Initiative and NASA's GEDI Mission Science Team. He has more than 60 peer-reviewed articles in international journals and of more than 300 contributions in international conferences and workshops.

He was awarded with the IEEE GRSS IGARSS Symposium Prize Paper Award in 1998, the Best Paper Award of the European SAR Conference (EUSAR) in 2002 and the IEEE GRSS J-STARS Paper Award in 2019. In 2002 he received the got the DLR science award, in 2011 he was awarded with DLR's Senior Scientist Award and with the IEEE Fellow Award in 2015.

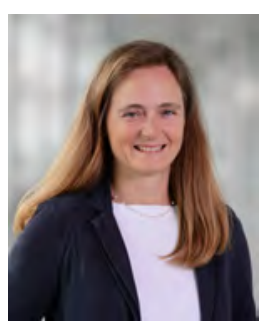

Irena Hajnsek (AM' 01, M'06, SM'09, FM'14) is since November 2009 Professor of Earth Observation at the Swiss Federal Institute of Technology (ETH) Zürich Institute of Environmental Engineering and at the same time head of the Polarimetric SAR Interferometry research group at the German Aerospace Center Microwaves and Radar Institute. Her main research interests are in electromagnetic propagation and scattering theory, radar polarimetry, SAR and interferometric SAR data processing techniques, environmental parameter modelling and estimation. She received her Dipl. degree (Honors) in 1996 from the Free University of Berlin, Germany and the Dr. degree (Honors) in 2001 from the Friedrich Schiller University of Jena, Germany. Since 2010 she is the science coordinator of the German satellite mission TanDEM-X and proposed satellite mission Tandem-L. She was Technical Program Co-chair of the IEEE IGARSS 2012 Symposium in Munich. Since 2013 she is a member of the IEEE GRSS AdCom and since 2016 she is the vice president of the IEEE GRSS Technical Committees. 